\title{
The Hierarchy of Equivalence Relations on the Natural Numbers Under Computable Reducibility ${ }^{*}$
}

\author{
Samuel Coskey \\ York University \& The Fields Institute \\ 222 College Street, Toronto, ON M5S 2N2, Canada \\ scoskey@nylogic.org \\ boolesrings.org/scoskey \\ Joel David Hamkins \\ Department of Philosophy, New York University \\ 5 Washington Place, New York, NY 10003 \& \\ The Graduate Center of the City University of New York \\ Mathematics Program, 365 Fifth Avenue, New York, NY 10016, \& \\ College of Staten Island of CUNY \\ 2800 Victory Blvd., Staten Island, NY 10314, USA \\ jhamkins@gc. cuny. edu \\ jdh.hamkins.org \\ Russell Miller \\ The Graduate Center of The City University of New York \\ Mathematics Program, 365 Fifth Avenue, New York, NY 10016 \& \\ Queens College of CUNY \\ 65-30 Kissena Blvd., Flushing, NY 11367, USA \\ Russell.Miller@qc. cuny. edu \\ qcpages.qc.cuny.edu/ rmiller
}

\begin{abstract}
The notion of computable reducibility between equivalence relations on the natural numbers provides a natural computable analogue of Borel reducibility. We investigate the computable reducibility hierarchy, comparing and contrasting it with the Borel reducibility hierarchy from descriptive set theory. Meanwhile, the notion of computable reducibility appears well suited for an analysis of equivalence relations on the c.e. sets, and more specifically, on various classes of c.e. structures. This is a rich context with many natural examples, such as the isomorphism relation on c.e. graphs or on computably presented groups. Here, our exposition extends earlier work in the literature concerning the classification of computable structures. An abundance of open questions remains.
\end{abstract}

Keywords: equivalence relations, computable reducability

\section{Introduction}

In this paper we aim to study the complexity of equivalence relations on the natural numbers and their hierarchy, under the relation of computable reducibility. Using a blend of methods from computability theory and descriptive set theory, we thus carry out a computable analogue of the theory of Borel equivalence relations. The resulting theory appears well suited for undertaking an analysis of the complexity of isomorphism relations and other equivalence relations on the class of computably enumerable (c.e.) structures, such as the isomorphism relation on c.e. graphs or on computably presented groups and the orbit equivalence relations arising from computable group actions. The c.e. analogues of many of the relations playing important roles in the Borel theory, such as equality, $E_{0}$ and others,

\footnotetext{
*The research of the first author is partially supported by the Natural Sciences and Engineering Research Council of Canada. The research of the second author has been partially supported by grants from the National Science Foundation, the Simons Foundation and the CUNY Research Foundation. The research of the third author has been partially supported by the Isaac Newton Institute and by grants from the National Science Foundation and the CUNY Research Foundation.
} 
play similar roles in the computable theory here, and in addition, the naturally arising equivalence relations from computability theory on c.e. sets, such as Turing equivalence, 1-equivalence and m-equivalence, also fit into the hierarchy. Although in broad strokes the resulting theory exhibits many of the important and attractive features of the Borel theory, there are notable differences, such as the existence of a complex hierarchy of relations strictly below the equality relation on c.e. sets, as well as relations incomparable to equality, and we shall remark on these differences when they arise.

In the subject known as Borel equivalence relations, one studies arbitrary equivalence relations on standard Borel spaces with respect to Borel reducibility, a notion introduced in [11]. Here, if $E, F$ are equivalence relations on $X, Y$, then we say that $E$ is Borel reducible to $F$, written $E \leq_{B} F$, if there is a Borel function $f: X \rightarrow Y$ such that

$$
x E x^{\prime} \Longleftrightarrow f(x) F f\left(x^{\prime}\right) .
$$

This notion is particularly meaningful when $E$ or $F$ represent a naturally arising classification problem in mathematics, for it allows us to compare the difficulty of such classification problems in a precise and robust manner. For instance, the isomorphism relations on the spaces of countable groups, graphs, fields, and linear orders, the isometry relation on separable Banach spaces, and the conjugacy relation on measure-preserving transformations can all be compared with respect to Borel reducibility. In these cases, we interpret $E \leq_{B} F$ as saying that the classification problem for elements of $X$ up to $E$ is no harder than the classification problem for elements of $Y$ up to $F$. Indeed, the reduction function $f$ yields a classification of the elements of $X$ up to $E$ using invariants from $Y / F$. For a fantastic introduction to the subject and its motivations we recommend the text [12], and we will often cite it for background material.

Motivated by the desire to imitate this field of research in the computable setting, we work with the following notion of reducibility for equivalence relations on the natural numbers.

Definition 1.1. Let $E, F$ be equivalence relations on $\mathbb{N}$. We say that $E$ is computably reducible (or just reducible) to $F$, written $E \leq F$, if there exists a computable function $f: \mathbb{N} \rightarrow \mathbb{N}$ such that

$$
n E n^{\prime} \Longleftrightarrow f(n) F f\left(n^{\prime}\right)
$$

In other words, the equivalence classes $\mathbb{N} / F$ form a set of effectively computable invariants for the classification problem up to $E$.

As evidence that this is a very natural definition, we remark that a substantial number of other authors have arrived at this notion from other directions. (Our own investigation of computable reducibility began independently from this prior work as well.) Without intending to give a completely history, we now briefly discuss this notion's travels through the literature. It seems that computable reducibility first appeared, at least in the English language, in [2], where it is defined only for c.e. equivalence relations. The authors of that paper note that the notion was in use prior to their work, for instance in [6]. The theory of c.e. equivalence relations with respect to computable reducibility was later expanded in [13]. More recently, and independently, Knight and coauthors considered a number of computability-theoretic notions of reducibility between various natural classes of structures (see the series of papers [3, 5, 14]). The notion of computable reducibility again arose in [7] and [9], where Fokina, Friedman and others used it to compare classes of computable structures. In [8], the authors used the notion to compare classes of hyperfinite structures, as well as arbitrary equivalence relations.

Meanwhile, the number of names given to the reducibility of Definition 1.1 is almost as large as the number of papers about it, and our name continues this tradition. The original name of $m$-reducibility, given by Bernardi and Sorbi and kept by Gao and Gerdes, reflects the analogy to $m$-reductions in computability theory (see e.g. [16]), but its use can be confusing, since one equivalence relation can be $m$-reducible to another in the sense of [2] yet not in the sense of [16], or vice versa. Knight and her coauthors actually used two distinct names for the same concept, since they viewed these as relations on classes of structures, not as reducibilities on equivalence relations. Finally, the notion is called FF-reducibility in [7-9]. We believe that computable reducibility serves our purposes best, since we are motivated by the analogy to Borel reducibility on equivalence relations. Borel reductions from $E$ to $F$ are 
Borel maps $f$ satisfying $x E y \Longleftrightarrow f(x) F f(y)$, and our reductions are Turing-computable maps $f$ with the same property. (We would gladly have called it "Turing reducibility," were that name not already in use.) Likewise, it would be natural to study $\boldsymbol{d}$-computable reducibility, using $\boldsymbol{d}$-computable functions $f$, for arbitrary Turing degrees $\boldsymbol{d}$, or to study $\mathcal{C}$-reducibility for other classes $\mathcal{C}$ of functions.

In this paper, we shall apply Definition 1.1 to the study of several varieties of equivalence relations. The simplest of these are the partitions of the natural numbers which are of a number-theoretic or combinatorial nature. Here, the notion of computable reducibility can be seen as a degree-theoretic structure on the equivalence relations which properly generalizes the classical Turing reducibilities. The connection with degree theory is more than just an analogy; for instance, we will observe in Proposition 2.6 that the 1-reducibility ordering on the c.e. 1-degrees embeds into the computable reducibility ordering on the equivalence relations with two classes.

Another connection with degree theory is the role of the arithmetical hierarchy. Here our theory departs from the classical Borel theory in a significant way. For instance, in the Borel theory almost all interesting equivalence relations are either Borel or $\Sigma_{1}^{1}$. As a consequence, equivalence relations cannot typically be distinguished up to Borel bireducibility just on the basis of their position in the projective hierarchy. In this paper, we shall consider equivalence relations on various levels of the arithmetic hierarchy, and this will give us a convenient and powerful tool for establishing nonreducibility.

A second, more substantial variety of equivalence relations, to which we shall devote most of our attention, are those arising from relations on the collection of computably enumerable (c.e.) subsets of $\mathbb{N}$. The goal is to study the hierarchy of equivalence relations arising naturally in this realm-including isomorphism relations on natural classes of c.e. structures, such as groups, graphs and rings-in a manner analogous to the Borel theory. Since the c.e. sets and structures have a canonical enumeration $\left\{W_{e}\right\}_{e \in \mathbb{N}}$ from computability theory, every equivalence relation on the c.e. sets arises from a corresponding equivalence relation on the indices for those sets, in effect using the program index $e$ to stand in for the set $W_{e}$ that it enumerates. Specifically, when $E$ and $F$ are equivalence relations defined on the c.e. sets, we can say that $E \leq F$ if and only if there is a computable function $f$ such that for all indices $e, e^{\prime}$,

$$
W_{e} E W_{e^{\prime}} \Longleftrightarrow W_{f(e)} F W_{f\left(e^{\prime}\right)} .
$$

Thus, $E \leq F$ in this sense if and only if $E^{c e} \leq F^{c e}$ in the sense of Definition 1.1, where $E^{c e}$ denotes the relation on $\mathbb{N}$ defined by $e E^{c e} e^{\prime} \Longleftrightarrow W_{e} E W_{e^{\prime}}$.

In this context, computable reducibility is more closely analogous with the Borel theory. For instance, many of the classically studied equivalence relations can be fruitfully restricted to just the c.e. sets. Moreover, many of the Borel reductions between these relations turn out to be computable in our sense, yielding a familiar hierarchy of equivalence relations on c.e. sets. As we shall see, there is even an analogue of the very important class of countable Borel equivalence relations.

The last type of equivalence relations that we shall consider are isomorphism relations, that is, equivalence relations which arise from classification problems. One might expect that we would be interested in the isomorphism relation on finite structures, since these are coded by natural numbers. However, it is computable whether two finite structures are isomorphic, and we shall see that computable equivalence relations are essentially trivial according to our reducibility notion. Instead, we shall consider isomorphism of c.e. structures by again using the indices as stand-ins for the structures they code. In this final context, our efforts either extend or stand in close analogy with the results in recent literature concerning effective notions of reducibility.

In addition to those already mentioned, many other important analogues of Borel reducibility theory appear in the literature. For instance the effective version of Borel reducibility, that is, hyperarithmetic reducibility between equivalence relations on the real or natural numbers, is studied in [10]. The even weaker notion of PTIME reducibility is considered in [1]. Finally, in [4] the authors consider a computability-theoretic strengthening of Borel reducibility, namely the reductions which are computable by an infinite time Turing machine.

This paper is organized as follows. In the next section we consider relations of the first variety, that is, relations on the natural numbers taken at face value. Here, we show that computable reducibility of equivalence relations in some sense generalizes both many-one and one-one reducibility of c.e. sets. In the third and fourth sections we 
begin to export some of the Borel equivalence relation theory to the theory of equivalence relations on c.e. sets, for instance observing that many of the classical Borel reductions hold in our context as well. On the other hand, we show that some unexpected phenomena occur, such as the existence of a large hierarchy of relations on c.e. sets which lie properly below the equality relation. In the fifth section we define and discuss c.e. analogues of the countable Borel equivalence relations and orbit equivalence relations. In the sixth section we introduce the theory of isomorphism and computable isomorphism relations on classes of c.e. structures. Finally, in the last section we compare equivalence relations arising from computability theory itself, such as the Turing degree relation on c.e. sets.

\section{Combinatorial Relations on $\mathbb{N}$}

In this section, we present a series of elementary results concerning relations on $\mathbb{N}$ of very low complexity. It should be noted that most of the statements here can be found in the aforementioned literature (see for instance [2, 13], and [8]).

We begin at what is for us the very lowest level, where some easy general observations lead quickly to a complete classification of the computable equivalence relations up to bireducibility. The fact that the computable relations are essentially trivial in the hierarchy of computable reducibility stands in contrast to the Borel theory, of course, where the Borel equivalence relations have a wildly rich structure under Borel reducibility. Meanwhile, at a level just above the computable relations, we show that the hierarchy immediately exhibits enormous complexity in the context of c.e. equivalence relations. Here, we mention just a few results; a much more detailed exposition of the relations at the c.e. level can be found in [13]. In later sections, we shall treat many natural equivalence relations arising from much higher realms of the arithmetic and even the descriptive set-theoretic hierarchies.

\section{Definition 2.1.}

- For each $n$ let $={ }_{n}$ be the equality relation on $0, \ldots, n-1$. In order to make the relation defined on all of $\mathbb{N}$, we throw the remaining numbers $n, n+1, \ldots$ into the equivalence class of $n-1$.

- Let $=_{\mathbb{N}}$ denote the equality relation on $\mathbb{N}$.

Thus $={ }_{n}$ is a canonically defined computable equivalence relation on $\mathbb{N}$ with exactly $n$ equivalence classes. Similarly, $\mathbb{N}$, is a canonical computable equivalence relation with infinitely many classes.

\section{Proposition 2.2.}

- If $E$ is any equivalence relation with at least $n$ classes, then $=_{n}$ is reducible to $E$.

- If $E$ is $\Pi_{1}^{0}$ and $E$ has infinitely many equivalence classes, then $=_{\mathbb{N}}$ is computably reducible to $E$.

Proof. Let $i_{0}, \ldots, i_{n-1}$ be a system of pairwise $E$-inequivalent natural numbers. Then $=_{n}$ is easily seen to be reducible to $E$ by the $\operatorname{map} f(k)=i_{k}$ for $k<n$, and $f(k)=i_{n-1}$ for $k \geq n$.

Now suppose that $E$ is $\Pi_{1}^{0}$ and that $E$ has infinitely many classes. Then $=_{\mathbb{N}}$ is reducible to $E$ by the map which, on input $n$, begins enumerating $E$-incomparable elements. When a system of $n$ pairwise $E$-incomparable elements is found, we map $n$ to the largest one.

On the other hand, we shall see later on that there exist c.e. equivalence relations which are computably incomparable with $=_{\mathbb{N}}$.

\section{Proposition 2.3.}

- If $E$ is a $\Sigma_{1}^{0}$ or $\Pi_{1}^{0}$ equivalence relation with finitely many equivalence classes, then $E$ is computable.

- If $E$ is computable and has exactly $n$ classes, then $E$ is computably reducible to $={ }_{n}$.

- If $E$ is computable, then $E$ is computably reducible to $=_{\mathbb{N}}$.

Proof. Begin by letting $i_{1}, \ldots, i_{n}$ be a maximal system of pairwise $E$-inequivalent natural numbers. If $E$ is $\Sigma_{1}^{0}$, then given natural numbers $a, b$ we can decide whether $a E b$ as follows. Begin enumerating $E$-equivalent pairs until it is discovered that $a E i_{j_{1}}$ and $b E i_{j_{2}}$. Then $a E b$ if and only if $j_{1}=j_{2}$. 

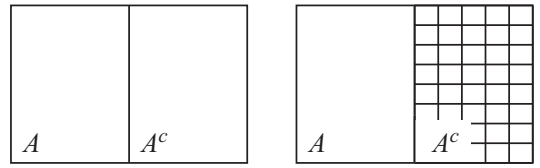

Figure 1. Left: The equivalence relations described in Definitions 2.5 (left) and 2.7 (right).

On the other hand, if $E$ is $\Pi_{1}^{0}$ then we can enumerate $E$-inequivalent pairs until we find that $a \not E i_{j}$ for all $j$ other than some $j_{1}$, and $b E i_{j}$ for all $j$ other than some $j_{2}$. Then again, $a E b$ if and only if $j_{1}=j_{2}$.

Finally, if $E$ is computable then given $a$, a program can order the equivalence classes which occur below $a$ by their least elements, and map $a \mapsto j$ if $a$ is in the $j^{\text {th }}$ class. Note that in the case that $E$ has just $n$ classes, then this is also a reduction to $=_{n}$.

We thus obtain a complete classification of the computable equivalence relations by the number of equivalence classes. This situation is identical to the classification of Borel equivalence relations with just countably many classes up to Borel reducibility.

Corollary 2.4. An equivalence relation $E$ is computable if and only if it is computably bireducible with one of $=_{n}$ or $=\mathbb{N}$.

The above results show that if we want to find distinct equivalence relations with some finite number of classes $n$, then we must look at least to the level $\Delta_{2}^{0}$. In fact, we need look no higher, since for instance any $\Delta_{2}^{0}$ noncomputable equivalence relation with exactly $n$ classes is not computably reducible to ${ }_{n}{ }_{n}$. We now observe that there is significant complexity even among the $\Delta_{2}^{0}$ equivalence relations with just two classes.

Definition 2.5. For $A \subseteq \mathbb{N}$, write $A^{c}$ for $\mathbb{N} \backslash A$. Then $E_{A, A^{c}}$ denotes the equivalence relation defined by $n E_{A, A^{c}} n^{\prime}$ if and only if both $n, n^{\prime} \in A$ or neither $n, n^{\prime} \in A$. That is, $E_{A, A^{c}}$ has two classes: $A$ and $A^{c}$.

It is clear that if $A$ is c.e., then the relation $E_{A, A^{c}}$ is $\Delta_{2}^{0}$; indeed, its complement is a difference of c.e. sets. The result below follows directly from the definition of many-one reducibility (see [16, Definition I.4.7]).

Proposition 2.6. $E_{A, A^{c}}$ is reducible to $E_{B, B^{c}}$ if and only if $A$ is many-one reducible to either $B$ or $B^{c}$.

Thus we obtain a copy of the partial ordering of many-one degrees, modulo the relation which identifies $A$ and $A^{c}$.

We now turn to a discussion of the c.e. equivalence relations. We show in particular that the computable reducibility hierarchy has an interesting and rich structure even at this low complexity level. The next result reflects the idea, hinted at in the introduction, that computable reducibility of equivalence relations in some sense generalizes the notion of one-one Turing reducibility of sets.

Definition 2.7. For any $A \subseteq \mathbb{N}$, let $E_{A}$ denote the equivalence relation defined by $n E_{A} n^{\prime}$ if and only if $n, n^{\prime} \in A$ or $n=n^{\prime}$. That is, $A$ is one equivalence class, and each remaining point is in an equivalence class by itself.

Observe that if $A$ is c.e., then $E_{A}$ is c.e.

Proposition 2.8. Suppose that $A, B \subseteq \mathbb{N}$ are c.e. and non-computable. Then $E_{A}$ is reducible to $E_{B}$ if and only if $A$ is 1-reducible to $B$.

Proof. If $f: \mathbb{N} \rightarrow \mathbb{N}$ is a 1-reduction from $A$ to $B$, then it is easy to see that $f$ is a also a computable reduction from $E_{A}$ to $E_{B}$. On the other hand, suppose that $f$ is a reduction from $E_{A}$ to $E_{B}$. Then $f(A)$ is either contained in $B$, or else it is a singleton. If $f(A)$ were a singleton, say $\{n\}$, then $A=f^{-1}(\{n\})$ would be computable, contradicting our hypothesis. Hence $f(A) \subseteq B$ and $f(\mathbb{N} \backslash A) \subseteq \mathbb{N} \backslash B$, and so $f$ is a many-one reduction from $A$ to $B$. Moreover, $f$ is already injective on $\mathbb{N} \backslash A$. 
Now, we will adjust $f$ on $A$ to obtain a 1-reduction $g$ from $A$ to $B$ as follows. Let $g(0)=f(0)$, and inductively let $g(n+1)=f(n+1)$ so long as $f(n+1)$ is distinct from $g(0), \ldots, g(n)$. On the other hand, if $f(n+1)=g(k)$ for some $k \in 0, \ldots, n$, then we must have that $f(n+1) \in B$. Hence we simply enumerate $B$ in search of a new element $a \in B$ which is distinct from $g(0), \ldots, g(n)$, and let $f(n+1)=a$. Then $g$ is as desired.

Thus the partial ordering of c.e. equivalence relations is at least as complicated as the 1-reducibility ordering on the c.e. 1-degrees. This last ordering is known to be quite complex. Moreover, we shall show later on that it contains a copy of the c.e. sets with the partial ordering of containment.

Corollary 2.9. There exist c.e. relations which are incomparable with $=_{\mathbb{N}}$.

Proof. Consider the relation $E_{A}$ where $A$ is a simple set (see [16, Theorem V.1.3]). That is, $A$ is a c.e. co-infinite set whose complement contains no infinite c.e. sets. Since $A$ is not computable, neither is $E_{A}$, and it follows that $E_{A}$ is not reducible to $=_{\mathbb{N}}$. On the other hand, if $f$ is a computable reduction from $=_{\mathbb{N}}$ to $E_{A}$ then there exists $n \in \mathbb{N}$ such that $f(\mathbb{N} \backslash\{n\}) \subseteq A^{c}$. But this is a contradiction, since $f(\mathbb{N} \backslash\{n\})$ is c.e. and infinite.

We next show that the class of c.e. equivalence relations, for all of its complexity, still admits a universal element. Again, this reflects the situation for the c.e. degrees.

Proposition 2.10. There exists a c.e. relation $U_{c e}$ which is universal in the sense that every c.e. equivalence relation is reducible to $U_{c e}$. Moreover, for any real parameter $z$, there exists an equivalence relation which is universal for all equivalence relations which are c.e. in $z$.

Proof. For any program $e$, let $E_{e}$ be the equivalence relation obtained by taking the transitive closure of whatever relation is enumerated by $e$, together with the diagonal for reflexivity. That is, we interpret the set $W_{e}$ as pairs, and we set $E_{e}$ to be the smallest equivalence relation containing these pairs. Thus, $E_{e}$ is the $e^{\text {th }}$ c.e. equivalence relation, and every c.e. equivalence relation arises this way.

Now define the universal relation by $(e, a) U_{c e}\left(e^{\prime}, a^{\prime}\right)$ if and only if $e=e^{\prime}$ and $a E_{e} a^{\prime}$. Thus, we have divided $\mathbb{N}$ into slices, and put $E_{e}$ on the $e^{\text {th }}$ slice. This relation is c.e., since we may computably enumerate approximations to $U_{c e}$ by running all programs and taking the transitive closure of what has been produced so far. It is universal for c.e. relations since $E_{e}$ reduces to $U_{c e}$ by mapping $a \mapsto(e, a)$.

This construction generalizes to oracles as follows. If $z$ is any oracle, we have the notion $E_{e}^{z}$ and we can define the relation $U_{c e}^{z}$ defined by performing the above construction relative to $z$. The relation $U_{c e}^{z}$ is $z$-c.e., and every $E_{e}^{z}$ computably reduces to $U_{c e}^{z}$ (without need for $z$ ) by the map $a \mapsto(e, a)$, as before.

Many of the results presented in this section so far are summarized in Figure 2.

We close this section with a result that is perhaps just a curiosity, but will motivate our discussion of orbit equivalence relations in later sections. In what follows, a group $\Gamma$ is said to be computable if its domain is $\mathbb{N}$ and its multiplication function is a computable set of triples. If $\Gamma$ is such a group, then a computable action of $\Gamma$ on $\mathbb{N}$ is just a computable function $\Gamma \times \mathbb{N} \rightarrow \mathbb{N}$ satisfying the usual group action laws. Finally, if $\Gamma$ acts computably on $\mathbb{N}$, then the resulting orbit equivalence relation is defined by $x E_{\Gamma} x^{\prime}$ if and only if there exists $\gamma \in \Gamma$ such that $x^{\prime}=\gamma x$.

Theorem 2.11. The c.e. equivalence relations are precisely the orbit equivalence relations induced by computable actions of computable groups.

Proof. Clearly if $E$ is the orbit equivalence relation induced by a computable action, then it is c.e. Conversely, suppose that $E$ is a c.e. relation, enumerated by a program $e$. Let $\Gamma$ be a fixed computable copy of the free group $F_{\omega}$ with generators $x_{1}, x_{2}, \ldots$ If $e$ enumerates a pair $\left(n, n^{\prime}\right)$ into $E$ at stage $s$, then we let $i$ be a code for the triple $\left(s, n, n^{\prime}\right)$ and let $x_{i}$ act by swapping $n$ and $n^{\prime}$ and leaving the other generators fixed. Clearly the orbit relation arising from this action is precisely $E$. Moreover the action is computable, since each generator codes a bound which tells how long to run $e$ to find out which elements it swaps. 


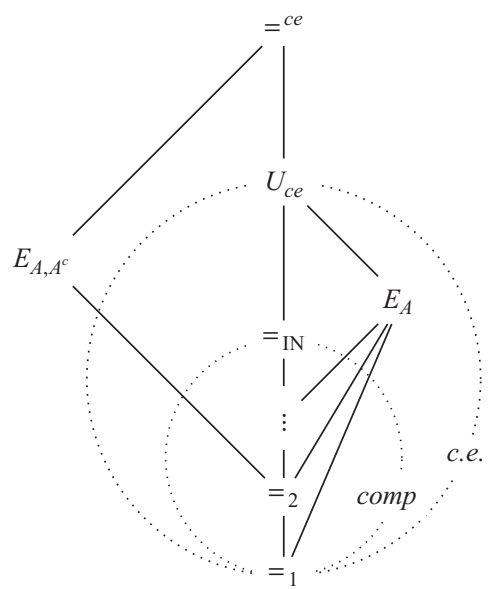

Figure 2. Diagram of reducibility among equivalence relations from Section 2 . The relation $={ }^{c e}$ is equality of c.e. sets as a relation on indices, namely, $e={ }^{c e} f \Longleftrightarrow W_{e}=W_{f}$, and it will be a major focus of section 3 .

\section{Equivalence Relations on c.e. Sets}

We now begin our study of the second variety of equivalence relations, namely, those defined on the c.e. real numbers. In this section, we will study a series of key equivalence relations from Borel relation theory. As was discussed in the introduction, the c.e. sets will be represented by their indices.

Definition 3.1. For any any equivalence relation denoted $E$ on the collection of c.e. subsets of $\mathbb{N}$, we shall denote by $E^{c e}$, adding the superscript " $c e$," the corresponding equivalence relation on $\mathbb{N}$ defined on the indices by $e E^{c e} e^{\prime}$ if and only if $W_{e} E W_{e^{\prime}}$.

One of the most important equivalence relation on c.e. sets is, of course, the equality relation.

Proposition 3.2. $={ }^{c e}$ is a $\Pi_{2}^{0}$-complete set of pairs.

Proof. To see that $={ }^{c e}$ is in $\Pi_{2}^{0}$, note that $W_{e}=W_{e^{\prime}}$ if and only if for all numbers $n$ enumerated into $W_{e}$, there is a stage by which $n$ is also enumerated into the other $W_{e^{\prime}}$ (and vice versa). To show that it is $\Pi_{2}^{0}$ complete, we use the fact that the set TOT of programs which halt on all inputs is $\Pi_{2}^{0}$ complete (see [16, Theorem IV.3.2]). The set TOT is $m$-reducible to the $={ }^{c e}$-equivalence class of $W_{e_{0}}=\mathbb{N}$ by the following function: $f(e)$ is the program which outputs $n$ just in case $e$ halts on input $n$.

Proposition 3.3. If $E$ is a c.e. equivalence relation, then $E$ lies properly below $={ }^{c e}$.

Proof. Let $E$ be an arbitrary c.e. relation. Since each equivalence class $[n]_{E}$ of $E$ is c.e., we can define a reduction from $E$ to ${ }^{c e}$ by mapping each $n$ to a program $f(n)$ which enumerates $[n]_{E}$. On the other hand, there can't be a reduction from $={ }^{c e}$ to $E$ since $={ }^{c e}$ is $\Pi_{2}^{0}$ complete and $E$ is c.e.

A second equivalence relation which plays an essential role in the Borel theory is the almost equality relation on $\mathcal{P}(\mathbb{N})$. Let $E_{0}$ denote this relation, that is, $A E_{0} B$ if and only if the symmetric difference $A \triangle B$ is finite. Then $E_{0}^{c e}$ is the almost equality relation on the (indices for) c.e. sets. We have the following analogue with the Borel theory.

Theorem 3.4. $={ }^{\text {ce }}$ lies strictly below $E_{0}^{c e}$.

Proof. It is not difficult to build a reduction from $={ }^{c e}$ to $E_{0}^{c e}$. For instance, given a program $e$, we can define the program $f(e)$ as follows. Whenever $n$ is enumerated into $W_{e}$, the program $f(e)$ enumerates codes for the pairs 
$(n, 0),(n, 1),(n, 2), \ldots$ (or more accurately, it arranges to periodically add more and more of these). Then clearly we have that $W_{e}$ and $W_{e^{\prime}}$ differ if and only if $W_{f(e)}$ and $W_{f\left(e^{\prime}\right)}$ differ infinitely often.

On the other hand, there cannot be a computable reduction from $E_{0}^{c e}$ to $={ }^{c e}$, since $E_{0}^{c e}$ is $\Sigma_{3}^{0}$ complete while $={ }^{c e}$ is just $\Pi_{2}^{0}$. To see that $E_{0}$ is $\Sigma_{3}^{0}$ complete, note that by [16, Corollary IV.3.5], even its equivalence class COF $=$ $\left\{e \mid W_{e}\right.$ is cofinite $\}$ is $\Sigma_{3}^{0}$ complete.

It is natural to wonder to what extent the arithmetic equivalence relations on c.e. sets mirror the structure of the Borel equivalence relations. For instance, it is a fundamental result of Silver (see [12, Theorem 5.3.5]) that the equality relation $=$ is minimum among all Borel equivalence relations with uncountably many classes. Thus, it is natural to ask whether an analogue of Silver's theorem holds, that is, whether $={ }^{c e}$ is minimum among some large class of relations on the c.e. sets with infinitely many classes. In the next section we shall show that this fails even for relations of very low complexity. However, we do not address any other analogues of the classical dichotomy theorems.

Question 3.5. Are there any relations lying properly between $={ }^{c e}$ and $E_{0}^{c e}$ ? More generally, is there a form of the Glimm-Effros dichotomy in this context? In other words, is there a large collection of equivalence relations $E$ such that if $={ }^{c e}$ lies strictly below $E$, then $E_{0}^{c e}$ lies below $E$ ?

We next consider some of the combinatorial equivalence relations that play key roles in the Borel theory.

\section{Definition 3.6.}

- Let $E_{1}$ be the equivalence relation on $\mathcal{P}(\mathbb{N})^{\mathbb{N}}$ defined by $\left(A_{n}\right) E_{1}\left(B_{n}\right)$ if and only if for almost all $n, A_{n}=B_{n}$.

- Let $E_{2}$ be the equivalence relation defined by $A E_{2} B$ if and only if $\sum_{n \in A \triangle B} 1 / n<\infty$.

- Let $E_{3}$ be the equivalence relation on $\mathcal{P}(\mathbb{N})^{\mathbb{N}}$ defined by $\left(A_{n}\right) E_{3}\left(B_{n}\right)$ if and only if for all $n, A_{n} E_{0} B_{n}$.

- Let $E_{\text {set }}$ be the equivalence relation on $\mathcal{P}(\mathbb{N})^{\mathbb{N}}$ defined by $\left(A_{n}\right) E_{\text {set }}\left(B_{n}\right)$ if and only if $\left\{A_{n} \mid n \in \mathbb{N}\right\}=\left\{B_{n} \mid n \in \mathbb{N}\right\}$.

- Let $Z_{0}$ denote the density equivalence relation defined by $A Z_{0} B$ if and only if $\lim |(A \triangle B) \cap n| / n=0$.

As usual, we are really interested in the corresponding "superscript c.e." relations on the indices. That is, we define $e E_{1}^{c e} e^{\prime}$ if and only if $W_{e}$ and $W_{e^{\prime}}$, thought of as subsets of the lattice $\mathbb{N} \times \mathbb{N}$, are identical in almost every column of this lattice. The relations $E_{3}^{c e}$ and $E_{\text {set }}^{c e}$ are defined analogously, using c.e. subsets of $\mathbb{N} \times \mathbb{N}$ to represent c.e. sequences of c.e. sets.

Proposition 3.7. The Borel reductions between the equivalence relations given in Definition 3.6 hold also in the case of computable reducibility. Specifically, $E_{0}^{c e}$ is reducible to $E_{1}^{c e}, E_{2}^{c e}$, and $E_{3}^{c e}$, and $E_{3}$ is reducible to $E_{\text {set }}$ and $Z_{0}$.

Sketch of proof. The reductions are just the same as the classical ones from the Borel theory. We will quickly give the reductions so that the reader may verify they are computable in our sense. To begin, notice that $E_{0}$ is reducible to $E_{3}$ by the map: $A \mapsto \mathbb{N} \times A$, where each column of the image of $A$ looks exactly like $A$ itself. To reduce $E_{0}$ to $E_{1}$, map $A$ to $\{\langle x, y\rangle \mid y \geq x \& y \in A\}$, so that the column $x$ equals $A-\{0, \ldots, x-1\}$. For $E_{0} \leq E_{2}$, we let $I_{n}$ be any fixed c.e. partition of $\mathbb{N}$ into sets such that $\sum_{i \in I_{n}} 1 / i \geq 1$. Then it is easy to see that $E_{0} \leq E_{2}$ via the map $A \mapsto \bigcup_{n \in A} I_{n}$.

To show that $E_{3} \leq Z_{0}$, we first observe that $E_{0}$ reduces to $Z_{0}$ via the map $f(A)=\bigcup_{n \in A}\left[2^{n}, 2^{n+1}\right.$ ). (Indeed, if $A E_{0}$ $B$ then $f(A) \triangle f(B)$ is finite and hence has density zero. Conversely, if $A E_{0} B$ is infinite, then $(f(A) \triangle f(B)) \cap N$ will be $\geq 1 / 2$ infinitely often, and hence $f(A) \triangle f(B)$ does not have density zero.) Now, fix a partition $I_{n}$ of $\mathbb{N}$ into sets such that the density of $I_{n}$ is exactly $1 / 2^{n}$, and let $\pi_{n}$ denote the unique isomorphism $\mathbb{N} \cong I_{n}$. Then we can reduce $E_{3}$ to $Z_{0}$ using the map $\left(A_{n}\right) \mapsto \bigcup \pi_{n}\left(f\left(A_{n}\right)\right)$.

Finally, to reduce $E_{3}$ to $E_{\text {set }}$, suppose we are given a sequence $\left(A_{n}\right)$. For each column $A_{n}$ and each $s \in 2^{<\mathbb{N}}$, we will place the set $1^{n} \frown{ }_{S} \frown(A \backslash|s|)$ as a column of $f\left(\left(A_{n}\right)\right)$. It is not difficult to check that this mapping is as desired. 

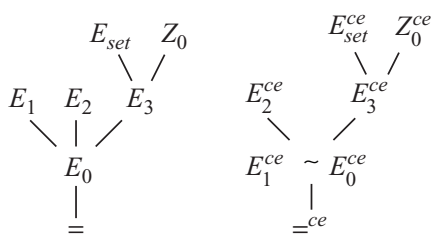

Figure 3. Left: The Borel reducibility relations between classical combinatorial equivalence relations. This diagram is complete for Borel reducibility. Right: Computable reducibility relations between c.e. versions of these relations. We do not know whether this diagram is complete for computable reducibility.

This result is summarized in Figure 3. We remark that the diagram is complete for Borel reducibility, in the sense that any edge not shown is known to correspond to a nonreduction. For computable reducibility, Theorem 3.8 uses the arithmetic hierarchy to show that the missing edges involving $E_{3}^{c e}$ remain nonreductions. The other missing edges are more difficult, and among them, the only one which we have settled appears further below as Theorem 3.10. Surprisingly, this theorem shows that $E_{1}^{c e} \leq E_{0}^{c e}$, which distinguishes the hierarchy for computable reducibility from that for Borel reducibility.

Theorem 3.8. $E_{3}^{c e}$ is not computably reducible to any of $E_{0}^{c e}$, $E_{1}^{c e}$ or $E_{2}^{c e}$.

Proof. We use a direct arithmetic complexity argument. Observe that $E_{0}^{c e}, E_{1}^{c e}$, and $E_{2}^{c e}$ are all easily seen to be $\Sigma_{3}^{0}$. On the other hand, we shall show that $E_{3}^{c e}$ is not $\Sigma_{3}^{0}$. In fact, we shall show that $E_{3}^{c e}$ has one equivalence class which is $\Pi_{3}^{0}$ complete, namely, the set $F$ consisting of indices $e$ for c.e. subsets of $\mathbb{N} \times \mathbb{N}$ such that every column of $W_{e}$ is finite. (As a matter of fact, $E_{3}^{c e}$ is $\Pi_{4}^{0}$-complete, but we will not prove this here.)

To begin, consider any set of the form $A=\{x \mid(\forall n)(\exists N)(\forall k) \phi(x, n, N, k)\}$, where $\phi$ is computable. For each $x$, we shall write down a program $e_{x}$ which enumerates a c.e. subset of $\mathbb{N} \times \mathbb{N}$ such that $x \in A$ if and only if $e_{x} \in F$. For each $n$, the program $e_{x}$ considers each $N$ in turn, and checks whether for all $k, \phi(x, n, N, k)$ holds. Whenever we find a counterexample $k$ for the current $N$, we enumerate one more element into the $n^{\text {th }}$ column of $W_{e_{x}}$. Observe that if $x \in A$, then for every $n$, we will eventually find the $N$ that works, and so the $n^{\text {th }}$ column of $W_{e_{x}}$ will be finite. Hence in this case $e_{x} \in F$. If $x \notin A$, then for some $n$, we will have to change our mind about $N$ infinitely often, and so the $n^{\text {th }}$ column of $W_{e_{x}}$ will be all of $\mathbb{N}$. Hence in this case $e_{x} \notin F$.

To prove that $E_{1}^{c e} \leq E_{0}^{c e}$, which surprised us, we will build the necessary computable reduction. This requires a detailed construction. To aid comprehension, we first present the basic module for this construction, stated here as proposition 3.9. Note that this proposition does not provide a reduction in the required sense, since the function $f$ depends on both $i$ and $j$; nevertheless, understanding this proof will help the reader follow the proof of the full Theorem 3.10.

Proposition 3.9. There exists a computable function $f$ such that, for every $i, j \in \omega$, we have

$$
W_{i} E_{1} W_{j} \Longleftrightarrow W_{f(i, j)} E_{0} W_{f(j, i)} .
$$

Proof. On inputs $i$ and $j$, let the output $f(i, j)$ be (a Gödel code for) the program which enumerates the set $W_{f(i, j)}$ we now describe. For each element $\langle c, k\rangle$ and each stage $s+1$, enumerate $\langle c, k\rangle$ into $W_{f(i, j), s+1}$ if the following hold:

- $\langle c, k\rangle \in W_{i, s} \backslash W_{j, s} ;$ and

- $(\forall n<k)\left[\langle c, n\rangle \in W_{i, s} \Longleftrightarrow\langle c, n\rangle \in W_{j, s}\right]$.

Additionally, if $\langle c, k\rangle \in W_{f(j, i), s} \cap W_{i, s} \cap W_{j, s}$, then enumerate $\langle c, k\rangle$ into $W_{f(i, j), s+1}$. This is the entire construction.

Now fix $c$, and suppose that $W_{i}$ and $W_{j}$ agree on their $c^{\text {th }}$ columns: $(\forall k)\left[\langle c, k\rangle \in W_{i} \Longleftrightarrow\langle c, k\rangle \in W_{j}\right]$. Then, if $\langle c, k\rangle$ ever entered $W_{f(i, j)}$, it did so either because it had already entered $W_{f(j, i)}$, or else because $\langle c, k\rangle \in W_{i, s} \backslash W_{j, s}$, in which case it must later have entered $W_{j}$ as well (by their agreement on the $c^{\text {th }}$ column), and therefore was then enumerated into $W_{f(j, i)}$ as well. Thus $W_{f(i, j)}$ and $W_{f(j, i)}$ agree with each other on their $c^{\text {th }}$ columns. 
On the other hand, suppose $W_{i}$ and $W_{j}$ disagree on their $c^{\text {th }}$ columns, and let $\langle c, k\rangle$ be the least point lying in just one of them. If $\langle c, k\rangle \in W_{i} \backslash W_{j}$, then by its minimality, it will eventually be enumerated into $W_{f(i, j), s+1}$ at some stage $s+1$. It will never appear in $W_{f(j, i)}$. Moreover, the only numbers $k^{\prime}$ such that $\left\langle c, k^{\prime}\right\rangle$ can be enumerated into either $W_{f(i, j)}$ or $W_{f(j, i)}$ at stages $>s+1$ are those which either sat in $W_{f(j, i), s+1}$ or $W_{f(i, j), s+1}$ already, or else have $k^{\prime}<k$, because once $\langle c, k\rangle$ has appeared in $W_{i, s+1}$, no $\left\langle c, k^{\prime}\right\rangle$ with $k^{\prime}>k$ could ever again be the least difference between the $c^{\text {th }}$ columns of $W_{i}$ and $W_{j}$. Therefore, $W_{f(i, j)}$ and $W_{f(j, i)}$ do differ on at least one element of their $c^{\text {th }}$ columns, but do not differ by more than finitely many elements. (In fact, each of these columns is finite.)

A symmetric argument holds when the least difference lies in $\left(W_{j}-W_{i}\right)$. Therefore, if $W_{i} E_{1} W_{j}$, then each of the finitely many columns on which they differ contains only finitely many differences between $W_{f(i, j)}$ and $W_{f(j, i)}$, and none of the other columns contain any differences at all between them. Thus $W_{f(i, j)} E_{0} W_{f(j, i)}$. Conversely, if $W_{i}$ and $W_{j}$ differ on infinitely many columns, then every one of those columns contains at least one difference between $W_{f(i, j)}$ and $W_{f(j, i)}$ as well, and so $W_{f(i, j)}$ and $W_{f(j, i)}$ are not $E_{0}$-related. Thus we have proven Proposition 3.9.

Adapting this basic module to cover all sets $W_{e}$ at once, rather than just two particular sets $W_{i}$ and $W_{j}$, is challenging, but it can be done. In the following, we write $W_{e}^{c}=W_{e} \cap\{\langle c, n\rangle \mid n \in \omega\}$ for the $c^{\text {th }}$ column of any c.e. set $W_{e}$. Also, for each $c$, we will need infinitely many infinite subsets $C^{c j}$ of $\omega$ on which to execute our construction. To avoid confusion, we refer to these sets as slices of $\omega$, not as columns.

Theorem 3.10. $E_{1}^{c e} \leq E_{0}^{c e}$. Hence, $E_{1}^{c e}$ and $E_{0}^{c e}$ are computably bireducible.

Proof. We define a computable function $g$ implicitly by building the c.e. sets $W_{g(i)}$ uniformly for $i \in \omega$. Here we give some intuition for the construction, before presenting it in full. The slice $C^{c j}$ is dedicated to making sure that, if the set $W_{j}$ differs on column $c$ from all the sets $W_{i}$ with $i<j$, then there should be at least one difference between $W_{g(j)}$ and each $W_{g(i)}$. At each stage $s+1$ in the construction, we will consider all $c, j \in \omega$ and all $i<j$, and define two auxiliary elements: $m_{s}^{c i j}$, which represents the least element of the $c^{\text {th }}$ column $\omega^{c}$ which lies in the symmetric difference of $W_{i, s}$ and $W_{j, s}$, and $x_{s}^{c j} \in C^{c j}$, the element we are currently using to distinguish $W_{g(i)}$ from $W_{g(j)}$ on the slice $C^{c j}$. The former will converge to the least element of $\left(W_{i} \triangle W_{j}\right) \cap \omega^{c}$, or diverge if this set is empty. Each time it changes, the current $x_{s}^{c j}$ enters $W_{g(i)} \cap W_{g(j)}$ and a new $x_{s+1}^{c j}$ is chosen and added to $W_{g(j)}$. The same will happen occasionally on behalf of some $k>j$, as described below, but in the end, $W_{g(i)}$ and $W_{g(j)}$ will agree on the slice $C^{c j}$ if and only if $W_{i}$ and $W_{j}$ agree on the column $\omega^{c}$.

We also must ensure that $\left(W_{g(i)} \triangle W_{g(j)}\right) \cap C^{c j}$ is finite, since $W_{j}$ and some $W_{i}$ might be equal on all other columns (hence might be $E_{1}$-related). The $C^{c j}$ slice is not intended to differentiate any $W_{i}$ and $W_{i^{\prime}}$ with $i^{\prime}<i<j$; indeed, we want to have $W_{g(i)} \cap C^{c j}=W_{g\left(i^{\prime}\right)} \cap C^{c j}$ for all such $i$ and $i^{\prime}$, leaving it to $C^{c i}$ to differentiate between them if necessary. Moreover, if any $i<j$ has $W_{i}^{c}=W_{j}^{c}$, then we will let $C^{c i}$ do the job, and $W_{g(j)} \cap C^{c j}$ will be equal to $W_{g(k)} \cap C^{c j}$ for every $k$, so that we do not repeat the process of adding differences between the same sets.

A set $W_{k}$ with $k>j$ might equal $W_{j}$ on the $c^{\text {th }}$ column, or might equal some $W_{i}$ with $i<j$ there. In the former case we would want to build $W_{g(k)}$ to be equal on $C^{c j}$ to $W_{g(j)}$, but in the latter case we would want it equal to $W_{g(i)}$ instead - which could pose difficulties if $W_{g(j)} \neq W_{g(i)}$ on $C^{c j}$. Our solution is to use the least difference (if any) between $W_{i}^{c}$ and $W_{j}^{c}$ to guess which is the case. If this minimum element $m^{c i j}=\lim _{s} m_{s}^{c i j}$ lies in both $W_{j}$ and $W_{k}$, for instance, then we can be sure that $W_{k}^{c} \neq W_{i}^{c}$, and if similar outcomes hold for each minimum (for every $i<j$ ), then we will make $W_{g(k)}$ look like $W_{g(j)}$ on $C^{c j}$. On the other hand, if there is some minimum element which shows that $W_{k}^{c} \neq W_{j}^{c}$, then on this slice we will make $W_{g(k)}$ look like the sets $W_{g(i)}$ (which are the same on this slice for all $i<j$ ). We do this for only finitely many $k>j$, namely those $\leq c$, to avoid having to add infinitely many elements to the sets $W_{g(j)}$ and $W_{g(i)}$, for that could wipe out the difference we would like to build between those sets. For each single $k$, there are only finitely many values $j$ and columns $c$ for which this restriction will stop $W_{k}$ from being considered in the construction of $W_{g(k)}$ on $C^{c j}$; whenever either $j \geq k$ or $c>k, W_{k}$ will be used in that construction. On the finitely many slices $C^{c j}$ in which it was not considered, $W_{g(k)}$ will have only a finite difference from any other set $W_{g(n)}$ anyway, which will have no effect on the question of whether $W_{g(k)} E_{0} W_{g(n)}$.

For the slice $C^{c j}$, there are two basic outcomes possible. First, suppose some $i<j$ satisfies $W_{i}^{c}=W_{j}^{c}$. Then there will be infinitely many stages $s$ at which $m_{s+1}^{c i j}$ is either $\neq m_{s}^{c i j}$ or undefined (if the symmetric difference is empty at 
that stage). At every one of these stages, the current $x_{s}^{c j}$, which already sat in $W_{g(j)}$, will be added to every set $W_{g(k)}$ with $k \neq j$, and the newly chosen $x_{s+1}^{c j}$ will be added to $W_{g(j)}$. Since this happens infinitely often, we wind up with $C^{c j} \subseteq W_{g(k)}$ for every $k \in \omega$.

On the other hand, suppose every $i<j$ has $W_{i}^{c} \neq W_{j}^{c}$. Then every sequence $\left\langle m_{s}^{c i j}\right\rangle_{s \in \omega}$ will converge to a limit $m^{c i j}$, the minimum of $W_{i}^{c} \triangle W_{j}^{c}$. Once we reach a stage $s_{0}$ at which all $W_{i, s_{0}} \Upsilon_{m^{c i j}+1}=W_{i} \uparrow_{m^{c i j}+1}$ and also $W_{j, s_{0}} \uparrow_{m^{c i j}+1}=$ $W_{j} \uparrow_{m^{c j}+1}$ for all $i$, the values $m_{s}^{c i j}$ will never change again, and therefore will never cause $x_{s_{0}}^{c j}$ to enter any $W_{g(i)}$. The indices $k$ with $j<k \leq m$ (if there are any) may yet cause this element to enter the sets $W_{g(i)}$. There are only finitely many such $k$, however, and each one causes this to happen at no more than one stage after $s_{0}$ (namely, the unique stage $s+1$, if one exists, such that $W_{j, s}^{c}$ and $W_{k, s}^{c}$ agree on the set of minima $\left\{m^{c i j} \mid i<j\right\}$, but $W_{j, s+1}^{c}$ and $W_{k, s+1}^{c}$ fail to agree on that set). Therefore, in this second outcome, there will exist a limit $x^{c j}=\lim _{s} x_{s}^{c j}$, which will lie in $W_{g(j)}$, will not lie in any $W_{g(i)}$ with $i<j$, and will lie in $W_{g(k)}($ for $j<k \leq m)$ if and only if $W_{k}$ and $W_{j}$ contain exactly the same elements from the set of minima. Moreover, this $x^{c j}$ will be the only element of $C^{c j}$ on which $W_{g(j)}$ differs from any set $W_{g(k)}$. So in this case we have accomplished the goal of establishing a single difference on the slice $C^{c j}$ between $W_{g(i)}$ and $W_{g(j)}$ for each $i<j$, while not differentiating $W_{g(i)}$ from $W_{g\left(i^{\prime}\right)}$ on this slice for any $i^{\prime}<i<j$. The point of our treatment of the sets $W_{g(k)}$ with $j<k \leq m$ was discussed above, and will appear below in Lemma 3.13. (The sets $W_{g(k)}$ with $k>m$ agree on $C^{c j}$ with all $W_{g(i)}$ for $i<j$, and hence differ by at most the element $x^{c j}$ from $W_{g(j)}$.)

Now we give the formal construction. At stage 0 , every element $m_{0}^{c i j}$ is undefined, and $x_{0}^{c j}$ is the least element of the slice $C^{c j}$. We also enumerate $x_{0}^{c j}$ into the set $W_{g(j), 0}$ (which as yet contains nothing other than this element).

At stage $s+1$, for each fixed $j$ and $c$ and for every $i<j$, we find the least element $m_{s+1}^{c i j}$ (if any) of the symmetric difference $\left(W_{i, s}^{c} \triangle W_{j, s}^{c}\right)$. Suppose first that there exists either an $i<j$ such that this symmetric difference is empty, or an $i<j$ such that $m_{s+1}^{c i j} \neq m_{s}^{c i j}$. Then we enumerate the $x_{s}^{c j}$ into every slice $W_{g(k), s+1}$, (we shall see that it was already in $W_{g(j), s}$, define $x_{s+1}^{c j}$ to be the next-smallest element of $C^{c j}$, and enumerate this $x_{s+1}^{c j}$ into $W_{g(j), s+1}$.

On the other hand, suppose that for every $i<j, m_{s+1}^{c i j}$ and $m_{s}^{c i j}$ are defined and equal to each other. In this case we consider each $k$ with $j<k \leq c$. (If $j \geq c$, we do nothing.) For each such $k$ in turn, we ask whether the following fact holds at this stage $s+1$ :

$$
(\forall i<j)\left[m_{s}^{c i j} \in W_{k, s+1} \Longleftrightarrow m_{s}^{c i j} \in W_{j, s+1}\right],
$$

and also whether the same fact held at the preceding stage $s$ :

$$
(\forall i<j)\left[m_{s}^{c i j} \in W_{k, s} \Longleftrightarrow m_{s}^{c i j} \in W_{j, s}\right] .
$$

If for some $k$ the fact held at the preceding stage but fails to hold now, then $x_{s}^{c j}$ already lies in all such slices $W_{g(k), s}$, and in this case we enumerate $x_{s}^{c j}$ into every $W_{g(i), s+1}$ for every $i$, define $x_{s+1}^{c j}$ to be the next-smallest element of $C^{c j}$, and enumerate this $x_{s+1}^{c j}$ into $W_{g(j), s+1}$. If there is no such $k$, we keep $x_{s+1}^{c j}=x_{s}^{c j}$.

Finally, for every $k$ for which the fact holds at the current stage and failed to hold at the preceding stage, we enumerate into $W_{g(k), s+1}$ the element $x_{s+1}^{c j}$ chosen above. This completes the construction.

To show that the computable function $g$ defined by this construction actually constitutes a reduction from $E_{1}^{c e}$ to $E_{0}^{c e}$, we prove a series of lemmas.

Lemma 3.11. For every $c, j, m$, and $n$, the sets $W_{g(m)} \cap C^{c j}$ and $W_{g(n)} \cap C^{c j}$ differ on at most the element $x^{c j}=$ $\lim _{s} x_{s}^{c j}$, if this limit exists. If $W_{i}^{c}=W_{j}^{c}$ for some $i<j$, then $x^{c j}$ does not exist, and the two sets above are equal.

Proof. At each stage $s$, on the slice $C^{c j}$, these two sets differ on at most $x_{s}^{c j}$, which enters $W_{g(j)}$ at one stage, might possibly enter certain sets $W_{g(k)}$ with $j<k \leq m$ at a subsequent stage, and then enters all other sets $W_{g(m)}$ when and if a new $x_{s+1}^{c j}$ is chosen. No elements of $C^{c j}$ ever enter any of these sets except those chosen at some stage $t$ as $x_{t}^{c j}$. So the lemma is clear. 
Lemma 3.12. For all triples $n<m<c$ with $W_{m}^{c} \neq W_{n}^{c}$ there is some $j \leq m$, the slice $C^{c j}$ has a limit element $x^{c j} \in$ $W_{g(n)} \triangle W_{g(m)}$.

Proof. If every $i<m$ satisfies $W_{i}^{c} \neq W_{m}^{c}$, then we simply take $j=m$, and the above analysis shows that $x^{c j} \in C^{c j}$ exists and lies in $W_{g(j)}$ (that is, in $\left.W_{g(m)}\right)$, but not in $W_{g(n)}$, since $n<j$.

Otherwise, choose the least $m^{\prime}<m$ with $W_{m^{\prime}}^{c}=W_{m}^{c}$ and the least $n^{\prime} \leq n$ with $W_{n^{\prime}}^{c}=W_{n}^{c}$. Let $i=\min \left(m^{\prime}, n^{\prime}\right)$ and $j=\max \left(m^{\prime}, n^{\prime}\right)$; thus $i<j$. (With $W_{n^{\prime}}^{c}=W_{n}^{c} \neq W_{m}^{c}=W_{m^{\prime}}^{c}$, we know $i \neq j$.) Now no $i^{\prime}<j$ satisfies $W_{j}^{c}=W_{i^{\prime}}^{c}$, so there exists a limit element $x^{c j}$ which lies in $W_{g(j)}$ but not in $W_{g(i)}$.

Suppose first that $W_{m}^{c}=W_{j}^{c}$. Then we have $j \leq m<c$, and so eventually $W_{m}$ and $W_{j}$ agree on all minima $m^{c i^{\prime} j}$, for all $i^{\prime}<j$. At every subsequent stage, $W_{g(m)} \cap C^{c j}$ will equal $W_{g(j)} \cap C^{c j}$, so in particular $x^{c j} \in W_{g(m)}$. Now if $n \leq j$, then $n<j$ because $W_{j}^{c}=W_{m}^{c} \neq W_{n}^{c}$ and so automatically $x^{c j}$ will not lie in $W_{g(n)}$. On the other hand, if $j<n$, then with $W_{n}^{c}=W_{i}^{c}$ and $i<j, m^{c i j}$ must lie in $W_{n}^{c} \triangle W_{j}^{c}$. Once $m^{c i j}$ has appeared in one of these sets, $W_{g(n)}$ will fail to contain $x_{s}^{c j}$ at all subsequent stages $s$, and therefore will fail to contain the limit $x^{c j}$. Thus $x^{c j}$ lies in $W_{g(m)} \backslash W_{g(n)}$, giving the difference we desired.

The exact same argument holds if $W_{n}^{c}=W_{j}^{c}$, only with $m$ and $n$ reversed. So we have proven the lemma.

We can now establish one direction of Theorem 3.10, namely that if $W_{m} Z_{1} W_{m}$ then $W_{g(m)} Z_{0} W_{g(n)}$. Indeed, if $W_{m}$ and $W_{n}$ differ on infinitely many columns $c$, then they differ on infinitely many columns $c$ with $c>\max (m, n)$. For each such $c$, Lemma 3.12 gives a $j$ and an element $x^{c j} \in C^{c j}$ on which $W_{g(m)}$ and $W_{g(n)}$ differ, as desired. It remains to prove the converse, namely that if $W_{m} E_{1} W_{n}$ then $W_{g(m)} E_{0} W_{g(n)}$. To begin with, we consider the columns $c$ on which they agree.

Lemma 3.13. Fix $m<n$ and $c \geq n$. If $W_{m}^{c}=W_{n}^{c}$, then for all $j \in \omega$ we have $W_{g(m)} \cap C^{c j}=W_{g(n)} \cap C^{c j}$.

Proof. Of course, if $W_{j}^{c}=W_{i}^{c}$ for some $i<j$, then every $W_{g(k)}$ contains all of $C^{c j}$, and we are done. So assume that there exists no such $i<j$, and that therefore the limit $x^{c j}$ is defined. We consider all possible values of $j$ relative to $m$ and $n$.

If $j=n$, then there is an $i<j$ (namely $i=m$ ) with $W_{i}^{c}=W_{j}^{c}$, and so $W_{g(n)} \cap C^{c j}=W_{g(m)} \cap C^{c j}=C^{c j}$.

If $j=m$, then $W_{n}^{c}$ agrees with $W_{j}^{c}$ on all minima $m^{c i j}$ with $i<j$, and so $W_{g(j)}$ and $W_{g(n)}$ both contain $x^{c j}$, and therefore are equal on $C^{c j}$. (This uses the fact that $n \leq c$.)

If $j<m$, then $W_{m}^{c}$ and $W_{n}^{c}$ either both agree with $W_{j}^{c}$ on all minima, or both disagree with it on some particular minimum. In the former case, they both contain $x^{c j}$, while in the latter case they both omit it. Either way they both are equal, by Lemma 3.11 .

If $j>n$, then automatically $W_{g(m)} \cap C^{c j}=W_{g(n)} \cap C^{c j}$, because for all $i<j, W_{g(i)}$ contains exactly those elements of $C^{c j}$ which are $<x^{c j}$.

Finally, suppose $m<j<n$. If $W_{j}^{c} \neq W_{i}^{c}$ for every $i<j$, then in particular $W_{j}^{c} \neq W_{m}^{c}=W_{n}^{c}$. So $x^{c j}$ fails to lie in $W_{m}^{c}$ (since $m<j$ ) and also fails to lie in $W_{n}^{c}$ (because they differ on the minimum $m^{c m j}$ ). By Lemma 3.11, therefore, $W_{g(m)}$ and $W_{g(n)}$ agree on $C^{c j}$.

Lemma 3.14. Fix $m<n$, and assume $W_{m}^{c}=W_{n}^{c}$. If $W_{g(m)} \cap C^{c j} \neq W_{g(n)} \cap C^{c j}$, then $c<n$ and $j \leq n$, and the symmetric difference $\left(W_{g(m)} \cap C^{c j}\right) \triangle\left(W_{g(n)} \cap C^{c j}\right)$ is finite.

Proof. That $c<n$ follows from Lemma 3.13. Moreover, we know that $W_{g(i)} \cap C^{c j}=W_{g\left(i^{\prime}\right)} \cap C^{c j}$ for every $i<i^{\prime}<$ $j$, and if $n<j$, then this applies to $m$ and $n$. Finally, Lemma 3.11 shows that the symmetric difference of the two sets contains at most one element.

Lemma 3.15. Suppose $W_{m} E_{1} W_{n}$, and let the set $C$ be the union of all those $C^{c j}$ with $j \in \omega$ and $W_{m}^{c}=W_{n}^{c}$. Then $\left(W_{g(m)} \cap C\right)$ and $\left(W_{g(n)} \cap C\right)$ differ by at most finitely many elements.

Proof. By Lemma 3.14, the difference is contained within finitely many slices $C^{c j}$, and that difference is finite (in fact, at most a single element) on each of those finitely many $C^{c j}$. 
So, for $m<n$ with $W_{m} E_{1} W_{n}$, it remains to consider $W_{g(m)}$ and $W_{g(n)}$ on those slices $C^{c j}$ with $W_{m}^{c} \neq W_{n}^{c}$. There are only finitely many such $c$, but there are infinitely many corresponding $j$. However, all but finitely many of these $j$ satisfy $m<n<j$, and for all those $j$, we know that $W_{g(m)} \cap C^{c j}=W_{g(n)} \cap C^{c j}$. On the finitely many remaining sets $C^{c j}$, there may be a difference, but only a finite difference, by Lemma 3.11. Thus, whenever $W_{m} E_{1} W_{n}$, we must have $W_{g(m)} E_{0} W_{g(n)}$. This completes the proof of Theorem 3.10.

Using Proposition 3.7 we also conclude that $E_{1}^{c e} \leq E_{2}^{c e}$ and $E_{1}^{c e} \leq E_{3}^{c e}$. The remaining questions in establishing a version of Figure 3 for computable reducibility are whether $E_{2}^{c e} \leq E_{1}^{c e}$ and whether either $E_{s e t}^{c e}$ or $Z_{0}^{c e}$ is $\leq E_{3}^{c e}$.

\section{Below Equality}

In this section, we add to the collection of known equivalence relations on c.e. sets which either lie properly below $={ }^{c e}$, or else are incomparable with $={ }^{c e}$, in the computable reducibility hierarchy. This is a departure from the Borel theory, where Silver's theorem implies that $=$ is continuously reducible to every nontrivial Borel equivalence relation. On the other hand, the situation is not entirely unfamiliar, being similar to that for other very weak reducibility notions (see for instance [1] for the case of PTIME reducibility). Indeed, all computably enumerable equivalence relations $E$ on $\omega$ are computably reducible to ${ }^{c e}$, just by letting $W_{f(e)}=\{i:\langle e, i\rangle \in E\}$, and so the work done in [2] and [13] all takes place within this realm.

We begin with the following examples, each of which is a simple (but not c.e.) equivalence relation.

\section{Definition 4.1.}

- Let $e E_{\min }^{c e} e^{\prime}$ if and only if $\min \left(W_{e}\right)=\min \left(W_{e^{\prime}}\right)$ or $W_{e}=W_{e}^{\prime}=\varnothing$.

- Let $e E_{\max }^{c e} e^{\prime}$ if and only if either $W_{e}, W_{e^{\prime}} \neq \varnothing$ and $\max \left(W_{e}\right)=\max \left(W_{e^{\prime}}\right)$, or $W_{e}=W_{e^{\prime}}=\varnothing$, or $\left|W_{e}\right|=$ $\left|W_{e^{\prime}}\right|=\aleph_{0}$.

It is easy to see that these relations are computably reducible to $={ }^{c e}$. Indeed, to show $E_{\min }^{c e} \leq={ }^{c e}$, given a program $e$ we saturate $W_{e}$ upwards by letting $f(e)$ enumerate the set $\left\{n \in \mathbb{N} \mid \exists l \in W_{e}(l \leq n)\right\}$. Similarly, to show $E_{\max }^{c e} \leq={ }^{c e}$, we saturate downwards with the program $f(e)$ that enumerates the set $\left\{n \in \mathbb{N} \mid \exists l \in W_{e}(l \geq n)\right\}$. In both cases, these functions are computable selectors, in the sense that $W_{f(e)}$ is $E_{\min }^{c e}$ or $E_{\max }^{c e}$ equivalent to $W_{e}$ and constant on these classes.

Remark 4.2. It is worth mentioning that $E_{\min }^{c e}$ and $E_{\max }^{c e}$ each admit another simple description. Namely, $E_{\min }^{c e}$ is computably bireducible with the relation $e E_{\mathrm{gcd}}^{c e} e^{\prime}$ if and only if $\operatorname{gcd}\left(W_{e}\right)=\operatorname{gcd}\left(W_{e^{\prime}}\right) .(\operatorname{Here} \operatorname{gcd}(S)$ is the greatest $n$ which divides every element of $S$, with $\operatorname{gcd}(\varnothing)=\infty$.) Indeed, to show $E_{\min }^{c e} \leq E_{\mathrm{gcd}}^{c e}$, we let $W_{f(e)}=\left\{n ! \mid n \in W_{e}\right\}$; while to show $E_{\mathrm{gcd}}^{c e} \leq E_{\min }^{c e}$, we let $W_{f(e)}=\left\{d<\infty \mid \exists s\left(d=\operatorname{gcd}\left(W_{e, s}\right)\right)\right\}$. Similarly, $E_{\max }^{c e}$ is bireducible with the relation $e E_{\mathrm{lcm}}^{c e} e^{\prime}$ if and only if $\operatorname{lcm}\left(W_{e}\right)=\operatorname{lcm}\left(W_{e^{\prime}}\right)$.

The next result gives our first example of a violation of Silver's theorem in the computable context.

Theorem 4.3. $E_{\max }^{c e}$ is not computably reducible to $E_{\min }^{c e}$. Consequently, $E_{\min }^{c e}$ lies properly below $={ }^{c e}$.

Proof. This holds for the simple reason that $E_{\min }^{c e}$ is $\Delta_{2}^{0}$, while $E_{\max }^{c e}$ is $\Pi_{2}^{0}$ complete. To see that $E_{\min }^{c e}$ is $\Delta_{2}^{0}$, observe that $W_{e}$ and $W_{e^{\prime}}$ have the same minimum if and only if for every $n$ in $W_{e}$ there exists an $m \leq n$ in $W_{e^{\prime}}$ and vice versa; and also if and only if there exists $n$ in $W_{e}$ and $W_{e^{\prime}}$ such that every $m \leq n$ is in neither $W_{e}$ nor $W_{e^{\prime}}$ (or both are empty). To see that $E_{\max }^{c e}$ is $\Pi_{2}^{0}$ complete, note that by [16, Theorem IV.3.2], even the $E_{\max }$ class INF $=\left\{e:\left|W_{e}\right|=\aleph_{0}\right\}$ is $\Pi_{2}^{0}$ complete.

In fact, $E_{\max }^{c e}$ and $E_{\min }^{c e}$ are incomparable up to computable reducibility, and hence both lie properly below $={ }^{c e}$. However, the proof that $E_{\min }^{c e}$ is not computably reducible to $E_{\max }^{c e}$ is slightly more difficult. For this, we require the monotonicity lemma, a key result which will be used a number of times over the next few sections. Before stating it, we need the following terminology. 


\section{Definition 4.4.}

- We say that $f: \mathbb{N} \rightarrow \mathbb{N}$ is well-defined on c.e. sets if $W_{e}=W_{e^{\prime}}$ implies $W_{f(e)}=W_{f\left(e^{\prime}\right)}$ (in other words, $f$ is a homomorphism from $={ }^{c e}$ to $={ }^{c e}$ ).

- We say that such a function $f$ is monotone if $W_{e} \subseteq W_{e^{\prime}}$ implies $W_{f(e)} \subseteq W_{f\left(e^{\prime}\right)}$.

Lemma 4.5 (Monotonicity lemma). If $: \mathbb{N} \rightarrow \mathbb{N}$ is computable and well-defined on c.e. sets, then $f$ is monotone.

Proof. Fix $e, e^{\prime}$, and $x$ such that $W_{e} \subseteq W_{e^{\prime}}$, and $x \in W_{f(e)}$; we must show that $x \in W_{f\left(e^{\prime}\right)}$ as well. We shall use the recursion theorem to design an auxiliary program $p$ which knows its own index, and hence the index of $f(p)$. The program $p$ simulates both $f(p)$ and $e$, and at first $p$ behaves just like $W_{e}$. Of course, if $p$ were to continue in this manner forever, then we would have $W_{p}=W_{e}$ and since $f$ is well-defined on c.e. sets, we would have $W_{f(p)}=W_{f(e)}$. It follows that there is some stage by which $x$ appears in $W_{f(p)}$. Once this occurs, $p$ begins to simulate $e^{\prime}$ and mimic its behavior instead of that of $e$. This does not contradict the earlier behavior of $p$, since $W_{e} \subseteq W_{e^{\prime}}$. Thus in the end, we will have $W_{p}=W_{e^{\prime}}$, and hence $W_{f(p)}=W_{f\left(e^{\prime}\right)}$, since $f$ is well-defined on c.e. sets. But we also arranged that $x \in W_{f(p)}$ and therefore $x \in W_{f\left(e^{\prime}\right)}$, as desired.

We can now complete the proof that $E_{\min }^{c e}$ and $E_{\max }^{c e}$ are computably incomparable.

Theorem 4.6. $E_{\min }^{c e}$ is not computably reducible to $E_{\max }^{c e}$. Consequently, $E_{\max }^{c e}$ lies properly below $={ }^{c e}$.

Proof. Suppose to the contrary that $f$ is a reduction from $E_{\min }^{c e}$ to $E_{\max }^{c e}$. We first claim that we can assume, without loss of generality, that $f$ is well-defined on c.e. sets. Indeed let $g$ be the reduction from $E_{\max }^{c e}$ to $={ }^{c e}$ given just below Definition 4.1. Then clearly $g \circ f$ is well-defined on c.e. sets. Moreover since $g$ is a selector, that is, $g(e) E_{\max }^{c e} e$, we have that $g \circ f$ is again a reduction from from $E_{\min }^{c e}$ to $E_{\max }^{c e}$. Hence, we may replace $f$ with $g \circ f$ to establish the claim.

Now, for each $n$, let $e_{n}$ be a program enumerating $[n, \infty)$. Then the sets $W_{e_{n}}$ form a monotone decreasing sequence of sets. By the claim, the monotonicity lemma implies that $W_{f\left(e_{n}\right)}$ is also a monotone decreasing sequence of sets. Moreover, since the $\min \left(W_{e_{n}}\right)$ are all distinct and $f$ is a reduction, we must have that $\max \left(W_{e_{n}}\right)$ are all distinct. It follows that $\max \left(W_{e_{n}}\right)$ is a strictly decreasing sequence of natural numbers, which is a contradiction.

At the end of the section, we will give a broad generalization of this argument. Before doing so, we will use these ideas to find an equivalence relation on c.e. sets which is incomparable with $={ }^{c e}$.

Definition 4.7. Let $E_{\mathrm{med}}^{c e}$ denote the equivalence relation on c.e. sets defined by $e E_{\mathrm{med}}^{c e} e^{\prime}$ if and only if the sets $W_{e}$ and $W_{e^{\prime}}$ are both finite and have the same median (or are both empty or both infinite).

Proposition 4.8. $E_{\mathrm{med}}^{c e}$ lies between $E_{\max }^{c e}$ and $E_{0}^{c e}$ in the reducibility hierarchy.

Proof. First, $E_{\max }^{c e} \leq E_{\operatorname{med}}^{c e}$ by the function $f$ which saturates downwards, that is, such that $W_{f(e)}=$ $\left\{n \mid \exists m \in W_{e}(n \leq m)\right\}$. Next, $E_{\text {med }}^{c e}$ is reducible to $E_{0}^{c e}$ via the function $e \mapsto f(e)$ defined as follows. The program $f(e)$ simulates $e$, and at each stage of simulation computes the median $r_{s}$ of the stage $s$ approximation $W_{e, s}$. As long as $r_{s}$ does not change, $f(e)$ will enumerate multiples of $r_{s}$ into $W_{f(e)}$. Whenever $r_{s}$ does change, $f(e)$ fills in everything up to its current maximum and starting there enumerates multiples of the new $r_{s}$.

Now, if $W_{e}$ and $W_{e^{\prime}}$ both have median $r$, then by some stage the medians of $W_{e, s}$ and $W_{e^{\prime}, s}$ will have both stabilized at $r$. Hence both $W_{f(e)}$ and $W_{f\left(e^{\prime}\right)}$ will both eventually contain just the multiples of $r$. If $W_{e}$ and $W_{e^{\prime}}$ are both empty or both infinite, then $W_{f(e)}$ and $W_{f\left(e^{\prime}\right)}$ will both be empty or all of $\mathbb{N}$, respectively. Finally, if $W_{e}$ and $W_{e^{\prime}}$ have distinct medians, then $W_{e}$ and $W_{e^{\prime}}$ will disagree on an infinite set.

Theorem 4.9. $E_{\mathrm{med}}^{c e}$ is incomparable with both $E_{\min }^{c e}$ and $={ }^{c e}$ with respect to computable reducibility.

Proof. It suffices to show that $E_{\mathrm{min}}^{c e}$ is not reducible to $E_{\mathrm{med}}^{c e}$ and that $E_{\mathrm{med}}^{c e}$ is not reducible to $={ }^{c e}$. Suppose first that $f$ is a reduction from $E_{\text {med }}^{c e}$ to $={ }^{c e}$. Then $f$ is well-defined on c.e. sets, and therefore it is monotone. Consider the three sets $W_{e_{1}}=\{1\}, W_{e_{2}}=\{1,2\}$, and $W_{e_{3}}=\{0,1,2\}$. Since $W_{e_{1}} \subseteq W_{e_{2}}$ and $\operatorname{med}\left(W_{e_{1}}\right) \neq \operatorname{med}\left(W_{e_{2}}\right)$, we have 
$W_{f\left(e_{1}\right)} \subsetneq W_{f\left(e_{2}\right)}$. Since $W_{e_{2}} \subseteq W_{e_{3}}$ and $\operatorname{med}\left(W_{e_{2}}\right) \neq \operatorname{med}\left(W_{e_{3}}\right)$, we have $W_{f\left(e_{2}\right)} \subsetneq W_{f\left(e_{3}\right)}$. It follows that $W_{f\left(e_{1}\right)} \neq$ $W_{f\left(e_{3}\right)}$. But this contradicts that $f$ is a reduction, since $\operatorname{med}\left(W_{e_{1}}\right)=\operatorname{med}\left(W_{e_{3}}\right)$.

Next suppose that $f$ is a reduction from $E_{\min }^{c e}$ to $E_{\operatorname{med}}^{c e}$, which means that $W_{e} E_{\min } W_{e^{\prime}} \Longleftrightarrow W_{f(e)} E_{\text {med }} W_{f\left(e^{\prime}\right)}$. Fix any index $e_{0}$ for which $W_{e_{0}}$ is nonempty and $W_{f\left(e_{0}\right)}$ has finite median $r_{0}$. Let $N=2 r_{0}+2$ and choose a finite sequence of programs $e_{1}, e_{2}, \ldots, e_{N}$, for which $\min \left(W_{e_{0}}\right)<\min \left(W_{e_{1}}\right)<\min \left(W_{e_{2}}\right)<\cdots<\min \left(W_{e_{N}}\right)$ and each $r_{i}=\operatorname{med}\left(W_{f\left(e_{i}\right)}\right)$ exists and is finite. Such a sequence exists because there are infinitely many different possible minimums for $W_{e_{i}}$ and at most two of these minimums leads to $W_{f\left(e_{i}\right)}$ being empty or infinite; all the rest have finite medians. Note also that the $r_{i}$ are distinct. Consider now the program $e$ that begins by enumerating $\min \left(W_{e_{N}}\right)$ into $W_{e}$. Since so far this set has the same minimum as $W_{e_{N}}$, it must eventually happen that there is a stage $s_{N}$ at which $W_{f(e), s_{N}}$ has median $r_{N}$. At such a stage, let program $e$ now enumerate $\min \left(W_{e_{N-1}}\right)$ into $W_{e}$. Because $W_{e}$ at this stage has the same minimum as $W_{e_{N-1}}$, if we do not add anything more to $W_{e}$ then there must be a stage $s_{N-1}$ at which the median of $W_{f(e), s_{N-1}}$ becomes $r_{N-1}$. And when this occurs, let program $e$ enumerate $\min \left(W_{e_{N-2}}\right)$ into $W_{e}$, and so on. After $N$ iterations of this process, we have a set $W_{e}$ with the same minimum as $W_{e_{1}}$, and at some eventual stage $s_{1}$ the median of $W_{f(e), s_{1}}$ becomes $r_{1}$. When this occurs, finally, we enumerate $\min \left(W_{e_{0}}\right)$ into $W_{e}$, thereby ensuring that the minimum of $W_{e}$ is the same as that of $W_{e_{0}}$, and so the median of $W_{f(e)}$ must now eventually become $r_{0}$. But a careful examination of our construction will reveal that $W_{f(e)}$ has at least $N$ elements, that is, at least $2 r_{0}+2$ many, since at least one new element was added at each step of the process. But if $W_{f(e)}$ has this many elements, then it is impossible for it to have median $r_{0}$, which is a contradiction. Therefore, no such reduction $f$ exists.

The relationships expressed by the last two results are summarized in Figure 4.

It is natural to generalize the examples of $E_{\min }$ and $E_{\max }$ to try to find a large family of simple incomparable relations. There are many possibilities for doing so, and in the remainder of this section we shall consider just one: a generalization to arbitrary computable linear orders. This will enable us to find numerous equivalence relations which are incomparable and lie below $={ }^{c e}$, and therefore strengthen our denial of Silver's theorem for computable reducibility.

In what follows, if $L$ is a linear ordering with order relation $<_{L}$ and $W \subseteq L$, then we let cut $L(W)$ denote the Dedekind cut determined by $W$, that is,

$$
\operatorname{cut}_{L}(W)=\left\{l \in L \mid \exists w \in W\left(l<_{L} w\right)\right\} .
$$

Evidently, if $L$ is a computable linear ordering with domain $\mathbb{N}$, and $W$ is a c.e. subset of $\mathbb{N}$, then cut $_{L}(W)$ is c.e. as well.

\section{Definition 4.10.}

- For $L$ a computable linear ordering, let $E_{L}$ denote the same cut equivalence relation defined by $e E_{L} e^{\prime}$ if and only if $\operatorname{cut}_{L}\left(W_{e}\right)=\operatorname{cut}_{L}\left(W_{e^{\prime}}\right)$.

- Similarly, we define $H_{L}$ to be the same hull equivalence relation defined by $e H_{L} e^{\prime}$ if and only if the convex hull of $W_{e}$ in $L$ is the same as the convex hull of $W_{e^{\prime}}$ in $L$.

For any computable $L$, the relations $E_{L}$ and $H_{L}$ are both computably reducible to $={ }^{c e}$ (by mapping $W_{e}$ to the cut or the hull that it determines, respectively). Moreover, both $E_{L}$ and $E_{L^{*}}$ are computably reducible to $H_{L}$, where $L^{*}$

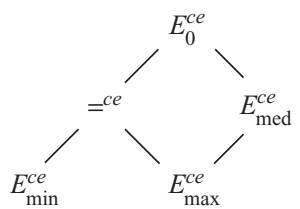

Figure 4. Diagram of reducibility among the relations in this section thus far. It follows from Theorems 4.3, 4.6, and 4.9 that this diagram is complete, in the sense that any edges not shown represent non-reducibilities. 
denotes the reverse of $L$. To see this, note that the cut map again defines a reduction $E_{L} \leq H_{L}$, and of course $H_{L}$ is bireducible with $H_{L^{*}}$, since in fact $H_{L}$ and $H_{L^{*}}$ are the same relation.

We have already seen several of the $E_{L}$ in another context. For instance, the relation $E_{\max }$ can be identified as $E_{\omega}$, where $\omega$ denotes the usual ordering on $\mathbb{N}$. Similarly, $E_{\min }$ can be identified with $E_{\omega^{*}}$. Finally, $={ }^{c e}$ is computably bireducible with $E_{\mathbb{Q}}$; to see that $={ }^{c e} \leq E_{\mathbb{Q}}$ consider the map which sends a c.e. set $W$ to the cut in $\mathbb{Q}$ corresponding to the real number $\sum_{n \in W} 1 / 3^{n+1}$.

We shall use the following notation. For $L$ a computable linear order, let $\bar{L}$ denote the set of c.e. cuts in $L$. We shall say that $\overline{L_{1}}$ is computably embeddable into $\overline{L_{2}}$, written $\overline{L_{1}} \hookrightarrow_{c} \overline{L_{2}}$, if there exists a computable function $\alpha: \mathbb{N} \rightarrow \mathbb{N}$ such that for all programs $e, e^{\prime}$, we have

$$
\operatorname{cut}_{L_{1}}\left(W_{e}\right)<\operatorname{cut}_{L_{1}}\left(W_{e^{\prime}}\right) \Longleftrightarrow \operatorname{cut}_{L_{2}}\left(W_{\alpha(e)}\right)<\operatorname{cut}_{L_{2}}\left(W_{\alpha\left(e^{\prime}\right)}\right) .
$$

The next result characterizes the structure of the $E_{L}$ relations with respect to computable reducibility.

Theorem 4.11. Let $L_{1}$ and $L_{2}$ be computable linear orders. Then $E_{L_{1}} \leq E_{L_{2}}$ if and only if $\overline{L_{1}} \hookrightarrow{ }_{c} \overline{L_{2}}$.

Proof. This is another application of the monotonicity lemma. Suppose first that $f$ is a computable reduction from $E_{L_{1}}$ to $E_{L_{2}}$. We can suppose without loss of generality that $f$ is well-defined on c.e. sets. (Indeed, simply postcompose $f$ with the map that sends $p$ to a program enumerating cut $_{L_{2}}\left(W_{p}\right)$.) It follows that $f$ is $\subseteq$-preserving and hence preserves the ordering on cuts. Moreover, since $f$ is a reduction, it must be injective on cuts. Hence it is an embedding of $\overline{L_{1}}$ into $\overline{L_{2}}$.

Next suppose that $\alpha: \overline{L_{1}} \hookrightarrow_{c} \overline{L_{2}}$. Then we simply define $W_{f(e)}=\operatorname{cut}_{L_{2}}\left(W_{\alpha(e)}\right)$, so that

$$
\begin{aligned}
e E_{L_{1}} e^{\prime} & \Longleftrightarrow \operatorname{cut}_{L_{1}}\left(W_{e}\right)=\operatorname{cut}_{L_{1}}\left(W_{e^{\prime}}\right) \\
& \Longleftrightarrow \operatorname{cut}_{L_{2}}\left(W_{\alpha(e)}\right)=\operatorname{cut}_{L_{2}}\left(W_{\alpha\left(e^{\prime}\right)}\right) \\
& \Longleftrightarrow W_{f(e)}=W_{f\left(e^{\prime}\right)} \\
& \Longleftrightarrow f(e) E_{L_{2}} f\left(e^{\prime}\right) .
\end{aligned}
$$

(The last equivalence holds because $W_{f(e)}$ and $W_{f\left(e^{\prime}\right)}$ are both cuts.) Hence, $f$ is a reduction from $E_{L_{1}}$ to $E_{L_{2}}$.

Remark 4.12. This result can also be generalized without much effort to the "same downwards closure" equivalence relation on c.e. subsets of computable partial orders.

Figure 5 elaborates on Figure 4 by showing the relationships between some sample $E_{L}$ which hold thanks to Theorem 4.11. Note that the reductions are strict as one moves to larger ordinals, because the cuts of a larger ordinal cannot map into the cuts of a smaller ordinal, and no infinite well-order can map into the cuts of its reverse. We end this section by mentioning a couple more easy consequences of this result.

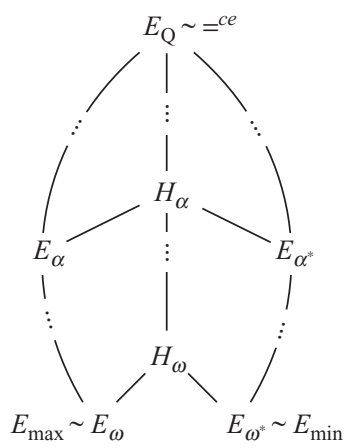

Figure 5. Diagram showing the cut and hull relations for computable ordinals $\alpha$ and their reverse orderings $\alpha^{*}$. In 2017 this will be the first diagram to land on Gliese $581 \mathrm{~g}$. 
Corollary 4.13. As long as $\bar{L} \hookrightarrow_{c} \overline{L^{*}}$, we have that $E_{L}$ and $E_{L^{*}}$ lie properly below $H_{L}$.

Corollary 4.14. There exist infinite chains and arbitrarily large finite antichains of equivalence relations on c.e. sets which lie below ${ }^{c e}$.

Sketch of proof. For instance, to construct an antichain of size three, consider computable copies of $L_{1}=\omega+\omega+$ $\omega^{*}, L_{2}=\omega+\omega^{*}+\omega$, and $L_{3}=\omega^{*}+\omega+\omega$. (For convenience, assume that all of the cuts are computable.) Then it is easy to check that for $i \neq j$ we have $\overline{L_{i}} \hookrightarrow \overline{L_{j}}$.

\section{Enumerable Equivalence Relations}

A great portion of Borel equivalence relation theory focuses on the countable Borel equivalence relations, that is, the Borel equivalence relations with every class countable. The foundation of this theory is the Lusin/Novikov theorem from descriptive set theory which states that every countable Borel equivalence relation can be enumerated in a Borel fashion. In other words, if $E$ is a countable Borel equivalence relation on $X$, then there exists a Borel function $f: X \rightarrow X^{\mathbb{N}}$ such that for all $x, f(x)$ enumerates $[x]_{E}$. Using this key fact, an argument of Feldman/Moore implies that any countable Borel equivalence relation can be realized as the orbit equivalence relation induced by a Borel action of a countable group. (See Theorem 7.1.2 and Theorem 7.1.4 of [12] for a discussion of these results.) In this section, we begin to develop a computable analogue of countable Borel equivalence relations.

We begin by introducing an analogue of the Lusin/Novikov property for equivalence relations on c.e. sets.

Definition 5.1. Let $E$ be an equivalence relation on c.e. sets. We say that $E^{c e}$ is enumerable in the indices if there exists a computable function $\alpha: \mathbb{N} \times \mathbb{N} \rightarrow \mathbb{N}$ such that $e E^{c e} e^{\prime}$ if and only if there exists $n \in \mathbb{N}$ such that $W_{\alpha(n, e)}=W_{e^{\prime}}$.

For example, $E_{0}^{c e}$ has this property. To see this, let $s_{n}$ denote the $n^{\text {th }}$ element of some computable enumeration of $2^{<\mathbb{N}}$, and fix a function $\alpha$ such that the program $\alpha(n, e)$ enumerates $s_{n} \frown\left(W_{e} \backslash\left|s_{n}\right|\right)$. Thus, as $n$ varies, the sets $W_{\alpha(e, n)}$ enumerates all finite modifications of $W_{e}$, and so $\alpha$ witnesses that $E_{0}^{c e}$ is enumerable in the indices.

Proposition 5.2. If $E^{c e}$ is enumerable in the indices then $E^{c e} \leq E_{\mathrm{set}}^{c e}$

Indeed, simply send a program $e$ to a program for a subset of $\mathbb{N} \times \mathbb{N}$ which acts like $\alpha(n, e)$ on the $n^{\text {th }}$ column. Of course $E_{\text {set }}^{c e}$ is not itself enumerable, since enumerable relations are easily seen to be $\Sigma_{3}^{0}$, whereas it follows from Theorem 3.8 that $E_{\text {set }}^{c e}$ is $\Pi_{3}^{0}$ complete.

We next consider the important special case of equivalence relations on c.e. sets which are induced, in an appropriate sense, by a computable action of a computable group.

Definition 5.3. Suppose that $\Gamma$ is a computable group acting on the c.e. subsets of $\mathbb{N}$. We say that the action is computable in the indices if there exists a computable function $\alpha: \mathbb{N} \times \mathbb{N} \rightarrow \mathbb{N}$ such that $W_{\alpha(\gamma, e)}=\gamma W_{e}$.

For example, if $\Gamma$ is a computable group then the left translation action of $\Gamma$ on the set $\mathcal{P}(\Gamma)^{c e}$ of c.e. subsets of $\Gamma$ is computable in the indices. We shall use the following notation: if the group $\Gamma$ acts on $C E$, which we write $\Gamma \curvearrowright C E$, then the induced orbit equivalence relation is defined by $e E_{\Gamma}^{c e} e^{\prime}$ if and only if $\exists \gamma \in \Gamma$ such that $W_{e^{\prime}}=\gamma W_{e}$.

One would like to prove an analogue of the Feldman/Moore theorem which would say that every enumerable relation is in fact the orbit relation of some action which is computable in the indices. Unfortunately, this is not the case. To get an idea of the difficulties involved, consider the natural action giving rise to $E_{0}$, namely, the bitwise addition action of $2^{<\mathbb{N}}$ on $\mathcal{P}(\mathbb{N})$. This action is highly effective in many natural senses, but not in the sense of this paper: given $e$ and $s \in 2^{<\mathbb{N}}$, it is not clear how to computably produce a program which enumerates the bitwise sum $s+W_{e}$. Indeed, the next result strongly negates the possibility that there is an analogue of Feldman/Moore in this context.

Theorem 5.4. Let $E$ be an equivalence relation on c.e. sets. Suppose that there exists $e \in \mathbb{N}$ such that $\left|\left[W_{e}\right]_{E}\right|>2$ and, for all $e^{\prime} E^{c e}$ e we have $W_{e} \subseteq W_{e^{\prime}}$. Then $E$ is not induced by any action which is computable in the indices. 
Proof. Suppose to the contrary that $\Gamma$ is a computable group acting on the c.e. sets and that $E$ is the induced orbit equivalence relation. Let $\alpha$ be the computable function which witnesses that the action of $\Gamma$ is computable in the indices. Then for each $\gamma$, the map $e \mapsto \alpha(\gamma, e)$ is well-defined on c.e. sets, and hence monotone. Now, choose $e^{\prime} E^{c e} e$ such that $W_{e} \subsetneq W_{e^{\prime}}$ and choose $\gamma \in \Gamma$ such that $\gamma W_{e^{\prime}}=W_{e}$. Now $\gamma W_{e} \subseteq \gamma W_{e^{\prime}}=W_{e}$, so by hypothesis, $\gamma W_{e}=W_{e}$ as well. This contradicts that $\alpha$ really gives rise to an action of $\Gamma$, since elements of a group must act by injective functions.

Corollary 5.5. $E_{0}^{c e}$ is not induced by any action which is computable in the indices.

Proof. The empty set $W_{e}=\varnothing$ is minimal in its $E_{0}$ equivalence class, which consists of all the finite sets.

This leaves open the following important question.

Question 5.6. Is $E_{0}^{c e}$ computably bireducible with an orbit relation induced by an action which is computable in the indices?

We close this section by showing that like the countable Borel equivalence relations, the orbit relations induced by actions which are computable in the indices admit a universal element. This gives some hope that the structure of the orbit equivalence relations on c.e. sets will mirror that of the countable Borel equivalence relations.

Proposition 5.7. There exists an equivalence relation $E_{\infty}^{c e}$ which is induced by an action that is computable indices, and satisfies $E_{\Gamma} \leq E_{\infty}^{c e}$ whenever $E_{\Gamma}$ arises from an action which is computable in the indices.

Proof. We begin by showing that for any computable group $\Gamma$ there exists an equivalence relation $U_{\Gamma}$ which is universal for equivalence relations induced by actions of $\Gamma$ which are computable in the indices. For this, we first regard c.e. sets as codes for functions $\phi: \Gamma \rightarrow C E$ (say by viewing them as subsets of $\Gamma \times \mathbb{N}$ and declaring $\phi(\gamma)=$ the $\gamma^{\text {th }}$ column). We then let $U_{\Gamma}$ be the equivalence relation induced by the action $(\gamma \cdot \phi)(g)=\phi\left(g \gamma^{-1}\right)$. It is easy to see that this action is computable in the indices. Moreover, if $E_{\Gamma}$ is the orbit relation induced by some other action of $\Gamma$ which is computable in the indices, then $E_{\Gamma}$ is reducible to $U_{\Gamma}$ via the function $f(e)=$ a program which enumerates the function $\phi_{e}: g \mapsto g W_{e}$. (Indeed, just check that $\gamma W_{e}=W_{e^{\prime}}$ if and only if $\gamma^{-1} \cdot \phi_{e}=\phi_{e^{\prime}}$.)

Now, let $F_{\omega}$ denote the free group on generators $x_{1}, x_{2}, \ldots$. We claim that we can take $E_{\infty}^{c e}$ to be $U_{F_{\omega}}$. To see this, let $E_{\Gamma}$ be the orbit equivalence relation induced by some action of $\Gamma$ which is computable in the indices. We can regard this as an action of $F_{\omega}$ by simply enumerating $\Gamma=\left\{\gamma_{i} \mid i \in \mathbb{N}\right\}$ and letting a word $w\left(x_{1}, \ldots x_{n}\right)$ act by the composition $w \circ\left(\gamma_{1}, \ldots, \gamma_{n}\right)$. It is easily seen that this action is computable in the indices, and so by the previous paragraph $E$ is computably reducible to $U_{F_{\omega}}$.

Figure 6 shows the two new classes defined in this section, and the handful of equivalence relations we have considered.

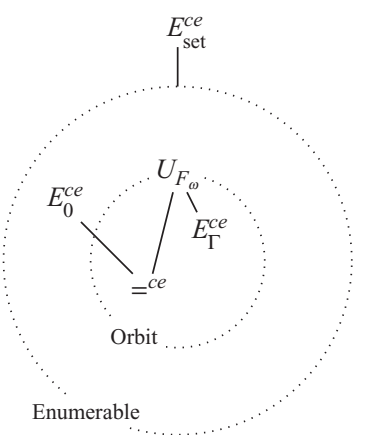

Figure 6. The enumerable relations. Note that we do not know whether $E_{0}$ is bireducible with an orbit equivalence relation. 
The results of this section have only scratched the tip of the iceberg. It would be very interesting to investigate the structure of the orbit equivalence relations on c.e. sets in further detail. For instance, we leave the following sample questions.

Question 5.8. Is $={ }^{c e}$ minimum among the relations induced by actions which are computable in the indices?

Question 5.9. Does there exist an infinite antichain of relations induced by actions which are computable in the indices?

\section{Classification of c.e. Structures}

We now direct our study towards what we expect will be one of the most important applications-isomorphism relations on classes of c.e. structures. As mentioned in the introduction, the isomorphism relations play a prominent role in Borel equivalence relations; in fact Borel reducibility was initially defined just for isomorphism relations on classes of countable structures. This has motivated several authors to consider various notions of computable reducibility between classes of countable structures. Here we begin to use the machinery built in earlier sections to study classes of c.e. structures.

Definition 6.1. Let $\cong_{\text {bin }}^{c e}$ denote the isomorphism relation on the codes for c.e. binary relations. That is, let $e \cong$ bin $e^{\prime}$ if and only if $W_{e}$ and $W_{e^{\prime}}$, thought of as binary relations on $\mathbb{N}$, are isomorphic.

We remark that in order to analyze the isomorphism on arbitrary $\mathcal{L}$-structures, it is enough to consider just the binary relations, since if $\mathcal{L}$ is a computable language then the isomorphism relation $\cong{ }_{\mathcal{L}}^{c e}$ on the c.e. $\mathcal{L}$-structures is computably reducible to $\cong$ bin. This follows from the proof of Proposition 6.2, cited below.

In analogy with the Borel theory, we can study the classification problem for c.e. undirected graphs, trees, linear orders, groups, and so on by considering the restriction of $\cong_{\text {bin }}^{c e}$ to the class of indices for such structures. Unfortunately, we immediately confront the difficulty that these restrictions are not total, and so far we have not addressed reducibility for relations which are not defined on all of $\mathbb{N}$.

In many practical situations, we can work around this difficulty. For instance, rather than identify graphs with binary relations on $\mathbb{N}$, we can identify them with subsets of a fixed computable copy $\Gamma$ of the random graph. Thus, we formally define $\cong_{\text {graph }}^{c e}$ to be isomorphism relation on the c.e. subsets of $\Gamma$. These two coding methods yield equivalent results in following sense: there is a computable reduction from $\cong_{\text {graph }}^{c e}$ to $\cong_{\text {bin }}^{c e}$ taking values in the indices for undirected graphs, and there is a computable function $f$ such that whenever $W_{e}, W_{e^{\prime}}$ code undirected graphs then $W_{e} \cong_{\text {bin }} W_{e^{\prime}}$ if and only if $W_{f(e)} \cong$ graph $W_{f\left(e^{\prime}\right)}$ (the last fact follows from Proposition 6.2 below). Similarly, we can consider the relation $\cong_{\text {lo }}^{c e}$ on the c.e. subsets of $\mathbb{Q}$, and $\cong_{\text {tree }}^{c e}$ on the downward closure of c.e. subsets of $\mathbb{N}<\mathbb{N}$.

Proposition 6.2. $\cong_{\text {bin }}^{c e}$ is computably bireducible with each of $\cong_{\mathrm{graph}}^{c e}, \cong_{10}^{c e}$, and $\cong_{\mathrm{tree}}^{c e}$.

The point is that the usual reductions go through in our context as well (see [12] for an elegant presentation). Intuitively, this is because the reductions only make use of the positive information about the structures; that is, they need to know when two elements are related, but not when two elements are non-related.

In terms of our hierarchy of equivalence relations, $\cong_{\text {bin }}^{c e}$ is very high. For one thing, it follows from results of [9] that it is $\Sigma_{1}^{1}$-complete. It also lies above most of the relations considered thus far; as an example we show the following:

Proposition 6.3. $E_{\text {set }}^{c e}$ lies properly below $\cong_{\text {bin }}^{c e}$.

Proof. To reduce $E_{\text {set }}^{c e}$ to $\cong$ bin, we simply let $W_{f(e)}$ be an code for $W_{e}$, thought of as a hereditarily countable set. In other words, $W_{f(e)}$ is a tree coding the transitive closure of $W_{e}$. The absence of any reverse reduction follows from complexity, since $E_{\text {set }}^{c e}$ is $\Pi_{4}^{0}$. 
We close our discussion of isomorphism relations by considering the case of groups. Classically, the isomorphism relation for countable groups is Borel bireducible with the other relations addressed in Proposition 6.2. However, in our case there are once again several possible coding methods. First, we can let group be the set of indices $e$ such that $W_{e}$, thought of as a subset of $\mathbb{N} \times \mathbb{N} \times \mathbb{N}$, satisfies the laws for the multiplication function for a group. We then define $\cong$ group to be the restriction of the isomorphism relation $\cong_{\text {tern }}^{c e}$ on the c.e. ternary relations to group. (Note that all of the elements of group are in fact computable, because they are c.e. functions.)

Alternatively we can code a group by a presentation, that is, a set of words in $F_{\omega}$, thinking of the group as the corresponding quotient. We thus let $e \cong$ pres $e^{\prime}$ if and only if $W_{e}$ and $W_{e^{\prime}}$, thought of as sets of relations in $F_{\omega}$, determine isomorphic groups. (Note that all groups with c.e. presentations actually have computable presentations by Craig's trick.) This relation has the advantage of being defined everywhere, but it does not reflect the same classification problem as $\cong$ group. In fact the classification problem for groups splits into two separate problems: that for computable group multiplication functions, and that for computably presented groups.

Proposition 6.4. $\cong_{\text {group }}^{c e} \leq \cong_{\text {bin }}^{c e} \leq \cong_{\text {pres }}^{c e}$.

We suspect that neither reduction is reversible.

Proof. Of course $\cong_{\text {group }}^{c e}$ is computably reducible to $\cong_{\text {tern }}^{c e}$ in the sense that there is a computable function (the identity) which, when restricted to group, satisfies $e \cong_{\text {group }}^{c e} e^{\prime}$ if and only if $f(e) \cong_{\text {tern }}^{c e} f\left(e^{\prime}\right)$. Hence, $\cong_{\text {group }}^{c e}$ is reducible to $\cong c e$ bin in the same sense. To see that $\cong_{\text {bin }}^{c e} \leq \cong_{\text {pres }}^{c e}$ one need only inspect the classical argument of [15], which yields group presentations, and check that it can be done in our context as well.

We next consider the computable isomorphism equivalence relations on these classes of structures.

Definition 6.5. Let $\simeq_{\text {bin }}^{c e}$ denote the computable isomorphism relation on the space of c.e. binary relations. That is $e \simeq_{\text {bin }}^{c e} e^{\prime}$ if and only if $W_{e}$ and $W_{e^{\prime}}$, thought of as codes for binary relations on $\mathbb{N}$, are isomorphic via a computable bijection.

The results of Proposition 6.2 apply also to the case of computable isomorphism. For instance if $\phi$ is a computable isomorphism between $W_{e}$ and $W_{e^{\prime}}$, and $f$ is the reduction given in [12] from binary relations to to graphs,

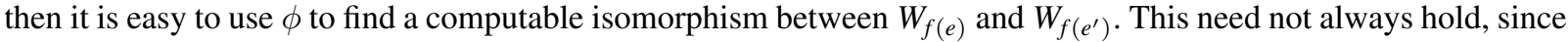
sometimes one is able to show that $W_{e} \cong W_{e^{\prime}}$ if and only if $W_{f(e)} \cong W_{f\left(e^{\prime}\right)}$ without necessarily constructing the isomorphisms explicitly. But it is not difficult to check that it does hold for the examples in this section.

Proposition 6.6. The computable isomorphism relation on the class of c.e. binary relations, graphs, linear orders and trees are all computably bireducible.

On the other hand, the computable isomorphism relation lies much lower in the hierarchy than the isomorphism relation.

Proposition 6.7. $\simeq_{\text {bin }}^{c e}$ lies properly below $E_{\mathrm{set}}^{c e}$.

Proof. Let $W_{e} \subseteq \mathbb{N}$ be a c.e. set, which we shall think of as coding a binary relation. We will create a c.e. subset $W_{f(e)}$ of $\mathbb{N} \cup-1 \times \mathbb{N}$ whose columns consist of all computably isomorphic copies of $W_{e}$ whose domain is a subset of $\mathbb{N}$, together with all finite sets of the form $F \cup-1$. To do this, $f(e)$ first arranges to write all finite sets of the form $F \cup-1$ onto the odd-numbered columns. Then, it considers all pairs of programs $p, p^{\prime}$, hoping in each case that $W_{p}$ codes a bijection $\phi_{p}$ of $\mathbb{N}$ with itself and $W_{p^{\prime}}$ codes its inverse. As $p$ is simulated, we write $\phi_{p}$ applied to the graph $W_{e}$ onto the column $2 n_{p, p^{\prime}}$ (where $n_{p, p^{\prime}}$ is a code for the pair $\left(p, p^{\prime}\right)$ ). If $W_{p}$ and $W_{p^{\prime}}$ do not turn out to code a bijection and its inverse then this will be apparent at some stage of simulation, and at that point we enumerate -1 into column $2 n_{p, p^{\prime}}$ and then stop writing to that column. It is not difficult to check that this reduction is as desired.

Finally, to see that $E_{\text {set }}^{c e}$ is not reducible to $\simeq_{\text {bin }}^{c e}$ simply compute that $\simeq_{\text {bin }}^{c e}$ is $\Sigma_{3}^{0}$, but it follows from Theorem 3.8 that in $E_{\text {set }}^{c e}$ there is a $\Pi_{3}^{0}$ complete equivalence class.

The hierarchy of isomorphism relations considered in this section is shown in Figure 7. 


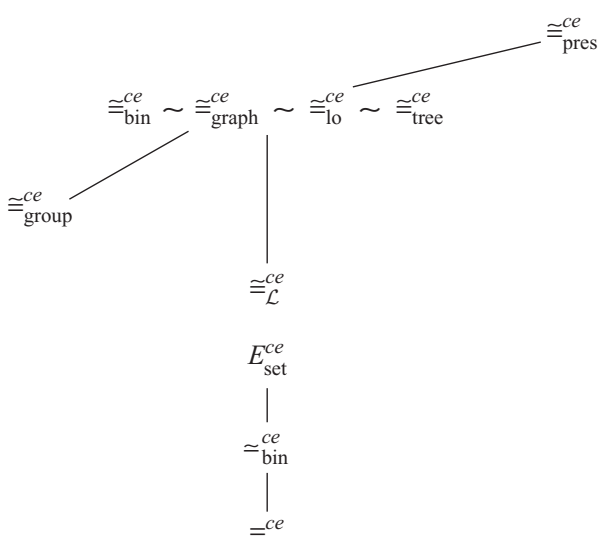

Figure 7. Relationships between the isomorphism equivalence relations considered in this paper.

\section{Relations from Computability Theory}

Some of the most important examples of relations on c.e. sets are those arising from computability theory itself. In this section, we consider the degree-theoretic equivalence relations, fitting them into the computable reducibility hierarchy. We then briefly generalize our method of dealing with the c.e. sets to handle the larger class of $n$-c.e. sets.

We begin with the degree-theoretic equivalence relations.

Theorem 7.1. $={ }^{c e}$ lies properly below each of $\equiv_{T}^{c e}, \equiv_{1}^{c e}$, and $\equiv_{m}^{c e}$ in the computable reducibility hierarchy.

Proof. We will define a function which reduces $={ }^{c e}$ to all three relations at once. To begin, we use a strong form of the Friedberg-Muchnik theorem to obtain a c.e. sequence of sets $A_{i}$ with the property that for all $i$, we have $A_{i} \not_{T} \bigoplus_{j \neq i} A_{j}$ (and also $\not_{1}$ and $\not \underline{L}_{m}$ ). Then, we let $W_{f(e)}$ be the subset of $\mathbb{N} \times \mathbb{N}$ whose $k^{\text {th }}$ column is $A_{n_{k}}$, where $n_{k}$ is the $k^{\text {th }}$ element enumerated into $W_{e}$ by $e$.

We first show that if $W_{e}=W_{e^{\prime}}$ then $W_{f(e)} \equiv_{1} W_{f\left(e^{\prime}\right)}$ (and hence $\equiv_{m}$ and $\equiv_{T}$ ). Assuming first that $W_{e}$ is infinite, $W_{f(e)}$ can be obtained from $W_{f\left(e^{\prime}\right)}$ by the following permutation of $\mathbb{N} \times \mathbb{N}$ : If the $k^{\text {th }}$ element to appear in $W_{e^{\prime}}$ is the $r^{\text {th }}$ element to appear in $W_{e}$, then send the $k^{\text {th }}$ column to the $r^{\text {th }}$ column. In the case that $W_{e}$ is finite, $W_{f(e)}$ can be obtained by $W_{f\left(e^{\prime}\right)}$ by a finite permutation of the columns.

We now show the converse: that if $W_{e} \neq W_{e^{\prime}}$ then $W_{f(e)} \not_{T} W_{f\left(e^{\prime}\right)}$ (and $\not_{1}$ and $\not_{m}$ ). For this, we can suppose that $i \in W_{e} \backslash W_{e^{\prime}}$. Now, suppose towards a contradiction that $W_{f(e)} \leq_{T} W_{f\left(e^{\prime}\right)}$. Then we have

$$
A_{i} \leq_{T} W_{f(e)} \leq_{T} \quad W_{f\left(e^{\prime}\right)} \leq_{T} \bigoplus_{j \neq i} A_{i},
$$

the last reduction holding using arguments similar to the previous paragraph. But this contradicts our choice of the $A_{i}$. As noted, this argument also works for $\leq_{1}$ and $\leq_{m}$.

Finally, none of the three degree relations are reducible to $={ }^{c e}$ because they are each $\Sigma_{3}^{0}$ complete (see for instance [16, Corollary IV.3.6]) while $={ }^{c e}$ is just $\Pi_{2}^{0}$.

Note that in defining the set $W_{f(e)}$ we cannot simply use the sum $\bigoplus_{j \in W_{e}} A_{j}$, since here a complicated set is coded into the indices of summation.

Theorem 7.2. $\equiv_{m}^{c e}$ is computably reducible to $\equiv_{1}^{c e}$.

Proof. For this we simply let $W_{f(e)}=W_{e} \times \mathbb{N}$. If $\phi: \mathbb{N} \rightarrow \mathbb{N}$ is a many-one reduction from $W_{e}$ to $W_{e^{\prime}}$, then the map $(m, n) \mapsto(\phi(m),\langle m, n\rangle)$ is a one-one reduction from $W_{f(e)}$ to $W_{f\left(e^{\prime}\right)}$. Conversely, if $\psi: \mathbb{N} \times \mathbb{N} \rightarrow \mathbb{N} \times \mathbb{N}$ is a 


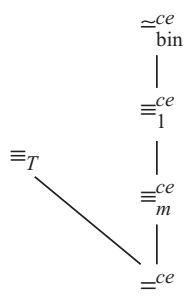

Figure 8. Relationships between the degree-theoretic equivalence relations and some others considered in earlier sections.

one-one reduction from $W_{f(e)}$ to $W_{f\left(e^{\prime}\right)}$, then the map $m \mapsto$ the first coordinate of $\psi(m, 0)$ is a many-one reduction from $W_{e}$ to $W_{e^{\prime}}$.

The next result clarifies how the degree-theoretic relations fit in with other relations previously considered. This and earlier results are depicted in Figure 8.

Proposition 7.3. $\equiv_{1}^{c e}$ is computably reducible to the computable isomorphism relation $\simeq_{\mathrm{bin}}^{c e}$ on c.e. binary relations.

Proof. The point is that $\equiv_{1}^{c e}$ is precisely the computable isomorphism relation on the set of c.e. unary relations. So for instance $\equiv_{1}^{c e} \leq \simeq_{\text {bin }}^{c e}$ via the map such that $W_{f(e)}$ codes the graph on $\mathbb{N} \cup\{\star\}$ where $\star \rightarrow n$ if and only if $n \in W_{e}$.

We close this section with a generalization of the reducibility hierarchy on c.e. sets. Here, we shall consider equivalence relations on the d.c.e. and even $n$-c.e. sets. This is natural given what we have done, because like the c.e. sets, the $n$-c.e. sets are also characterized by natural number indices.

\section{Definition 7.4.}

- If $\boldsymbol{e}=\left\langle e_{1}, \ldots, e_{n}\right\rangle$ is a sequence of indices then the corresponding $n$-c.e. subset of $\mathbb{N}$ is the set $W_{\boldsymbol{e}}=\left(W_{e_{1}}\right\rangle$ $\left.W_{e_{2}}\right) \cup\left(W_{e_{3}} \backslash W_{e_{4}}\right) \cup \cdots \backslash \cup W_{e_{n}}$. Here, the last operation is either $\backslash$ or $\cup$ depending on whether $n$ is even or odd.

- If $E$ is an equivalence relation on $n$-c.e. sets, then $E^{n-c e}$ is the relation on $\mathbb{N}^{n}$ defined by $\boldsymbol{e} E^{n-c e} \boldsymbol{f}$ if and only if $W_{e} E W_{f}$.

The 1-c.e. sets are of course the c.e. sets, and the 2-c.e. sets are sometimes called the d.c.e. sets (difference of c.e. sets). Thus we shall write $E^{d c e}$ for $E^{2-c e}$. It is trivial to check that for all $n$ we always have $E^{n-c e} \leq E^{n-c e} \leq E^{n+1-c e}$ : fix an $e$ with $W_{e}=\varnothing,\left(e_{1}, \ldots, e_{n}\right) \mapsto\left(e_{1}, \ldots, e_{n}, e\right)$.

Theorem 7.5. For every $n>0,={ }^{n-c e}$ lies properly below $={ }^{(n+1)-c e}$.

Proof. We claim that there is no computable reduction from $=^{(n+1)-c e}$ to $=^{n-c e}$. Suppose that $g$ were such a reduction. Fix some $\boldsymbol{e}$ and $\boldsymbol{f}$ of length $(n+1)$ with $W_{\boldsymbol{e}}=\{0\}$ and $W_{\boldsymbol{f}}=\varnothing$. By assumption, $W_{g(\boldsymbol{e})} \neq W_{g(\boldsymbol{f})}$, and we suppose first that there exists some number $m \in W_{g(e)} \backslash W_{g(f)}$. Of course, the $n$-c.e. sets $W_{g(\boldsymbol{e})}$ and $W_{g(f)}$ can be approximated using the indices $g(\boldsymbol{e})$ and $g(\boldsymbol{f})$. We will use the Recursion Theorem to define an $(n+1)$-c.e. set $W_{i}$ which "knows its own indices $i$ " and is approximated as follows. We will use the notation $W_{i, s}$ denote the approximation to $W_{i}$ at stage $s$.

We start with $W_{i, 0}=\varnothing$ and $W_{i, 1}=\{0\}$. At the first stage $s_{0}$ (if any) with $m \in W_{g(i), s_{0}}$, we take 0 out, leaving $W_{i, s_{0}+1}=\varnothing$. Then we do nothing further until the next stage $s_{1}>s_{0}$ at which $m \notin W_{g(i), s_{1}}$. At stage $s_{1}+1$, we enumerate 0 back into $W_{i}$, leaving $W_{i, t+1}=\{0\}$. Then, if we encounter another stage $s_{2}>s_{1}$ at which $m$ enters $W_{g(i)}$ again, we take 0 back out of $W_{i}$, and so on, back and forth, as many times as $m$ enters or leaves the set $W_{g(i)}$. This happens at most $n$ times, so $W_{i}$ is $(n+1)$-c.e. (including the initial enumeration of 0 into $\left.W_{i, 1}\right)$. However, our construction leaves $W_{i}=\varnothing=W_{f}$ if and only if $m \in W_{g(i)}$, in which case $W_{g(i)} \neq W_{g(f)}$; whereas, if $m \notin W_{g(i)}$, then $W_{i}=\{0\}=W_{\boldsymbol{e}}$, yet $W_{g(i)} \neq W_{g(\boldsymbol{e})}$, since $m \in W_{g(\boldsymbol{e})}$. 


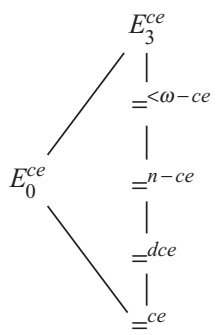

Figure 9. Known relationships between the relations on $n$-c.e. sets.

If there is no $m$ in $W_{g(\boldsymbol{e})} \backslash W_{g(\boldsymbol{f})}$, then there must be some number $m^{\prime} \in W_{g(\boldsymbol{f})} \backslash W_{g(\boldsymbol{e})}$, since $W_{\boldsymbol{e}} \neq W_{\boldsymbol{f}}$ and $g$ is assumed to be a reduction. In this case, we execute the same construction, except that we leave $W_{i, s}=\varnothing$ until reaching a stage $s_{0}$ with $m^{\prime} \in W_{g(i), s_{0}}$, then enumerate 0 into $W_{i}$, then wait for $m^{\prime}$ to leave $W_{g(i)}$, then remove 0 from $W_{i}$, and so on. In this case, $W_{i}$ is actually just $n$-c.e., not $(n+1)$-c.e., and again the strategy works: if $m^{\prime} \in W_{g(i)}$, then $W_{i}=\{0\}=W_{e}$, yet $m^{\prime} \notin W_{g(e)}$; whereas, if $m^{\prime} \notin W_{g(i)}$, then $W_{i}=\varnothing=W_{f}$, yet $m^{\prime} \in W_{g(f)}$. So $g$ cannot have been a computable reduction.

We can define an equivalence relation above all of these. Let $\boldsymbol{h}: \omega \rightarrow \omega^{<\omega}$ be a computable bijection, and define

$$
i \equiv{ }^{<\omega-c e} j \Longleftrightarrow W_{\boldsymbol{h}(i)}=W_{\boldsymbol{h}(j)} .
$$

Here, if $\boldsymbol{h}(i) \in \omega^{<\omega}$ has length $n$, then $W_{\boldsymbol{h}(i)}$ is exactly the $n$-c.e. set defined above. So this relation $=<\omega-c e$ is really just an amalgam of all the relations $={ }^{n-c e}$. It is clear that $=^{n-c e} \leq=<\omega-c e$ for every $n$, and Theorem 7.5 then shows that $=<\omega-c e \not \leq={ }^{n-c e}$.

Many of the results in this paper concerning equivalence relations on c.e. sets have analogues for the $n$-c.e. sets. However, it is also interesting to consider how relations on $n$-c.e. sets fit in with the relations on the c.e. sets.

Theorem 7.6. The relation $=<\omega-c e$ is computably reducible to $E_{3}^{c e}$, but no reduction exists in the opposite direction.

Proof. For the reduction, let $\boldsymbol{e}=\left\langle e_{1}, \ldots, e_{n}\right\rangle$ be an index for an $n$-c.e. set. We define a program $f\left(\left(e_{i}\right)\right)$ enumerating a subset of $\mathbb{N} \times \mathbb{N}$ as follows. Begin by simulating the programs $e_{1}, \ldots, e_{n}$. If $k$ appears in $W_{e, s}$, we write the first $s$ elements of the $k^{\text {th }}$ column into $W_{f(\boldsymbol{e})}$. Note that as $s$ increases, the status of $k \in W_{\boldsymbol{e}, s}$ can only change $n$ times, and therefore $k \in W_{\boldsymbol{e}}$ if and only if the $k^{\text {th }}$ column of $W_{f(e)}$ is infinite. It follows easily that $f$ is a reduction to $E_{3}^{c e}$.

In the opposite direction, we note that the relation $E_{3}^{c e}$ is $\Pi_{3}^{0}$-complete. However, $W_{e}=W_{f}$ iff, for every $x$, we have $x \in W_{\boldsymbol{e}}$ if and only if $x \in W_{\boldsymbol{f}}$. Since membership in each of $W_{\boldsymbol{e}}$ and $W_{\boldsymbol{f}}$ is $\Delta_{2}^{0}$, the relation $=<\omega-c e$ is $\Pi_{2}^{0}$, precluding any computable reduction.

These relationships are shown in Figure 9. It would also be interesting to decide the relationship between $=^{n-c e}$ and $E_{0}^{c e}$. Since $E_{0}^{c e}$ is is $\Sigma_{3}^{0}$ complete while $=^{n-c e}$ is just $\Pi_{2}^{0}$, we can conclude that $E_{0}^{c e}$ is not computably reducible to $={ }^{n-c e}$. This leaves open the following question.

Question 7.7. Are any of the $={ }^{n-c e}$ computably reducible to $E_{0}^{c e}$ ?

\section{References}

[1] S. Buss, Y. Chen, J. Flum, S.-D. Friedman, and M. Müller. Strong isomorphism reductions in complexity theory. Journal of Symbolic Logic, 76(4):1381-1402, 2011.

[2] C. Bernardi and A. Sorbi. Classifying positive equivalence relations. Journal of Symbolic Logic, 48(3):529$538,1983$. 
[3] W. Calvert, D. Cummins, J. F. Knight, and S. Miller. Comparing classes of finite structures. Algebra and Logic, 43(6):374-392, 2004.

[4] S. Coskey and J. D. Hamkins. Infinite time computable equivalence relations. Notre Dame Journal of Formal Logic, 52(2):203-228, 2011.

[5] W. Calvert and J. F. Knight. Classification from a computable viewpoint. The Bulletin of Symbolic Logic, 12(2):191-218, 2006.

[6] J.. L. Eršov. Teoriya numeratsii. "Nauka”, Moscow, 1977. Matematicheskaya Logika i Osnovaniya Matematiki. [Monographs in Mathematical Logic and Foundations of Mathematics].

[7] E. B. Fokina and S.-D. Friedman. Equivalence relations on classes of computable structures. In Proceedings of the 5th Conference on Computability in Europe: Mathematical Theory and Computational Practice. SpringerVerlag, 2009.

[8] E. B. Fokina and S.-D. Friedman. On $\Sigma_{1}^{1}$ equivalence relations over the natural numbers. Mathematical Logic Quarterly, 2011.

[9] E. B. Fokina, S.-D. Friedman, V. Harizanov, J. F. Kinght, C. McCoy, and A. Montalbán. Isomorphism and bi-embeddability relations on computable structures. Preprint, 2010.

[10] E. B. Fokina, S.-D. Friedman, and A. Törnquist. The effective theory of Borel equivalence relations. Annals of Pure and Applied Logic, 161(7):837-850, 2010.

[11] H. Friedman and L. Stanley. A Borel reducibility theory for classes of countable structures. Journal of Symbolic Logic, 54(3):894-914, 1989.

[12] S. Gao. Invariant Descriptive Set Theory. Pure and Applied Mathematics (Boca Raton). CRC Press, Boca Raton, FL, 2009.

[13] S. Gao and P. Gerdes. Computably enumerable equivalence relations. Studia Logica, 67(1):27-59, 2001.

[14] J. F. Knight, S. Miller, and M. V. Boom. Turing computable embeddings. Journal of Symbolic Logic, 72(3): 901-918, 2007.

[15] A. H. Mekler. Stability of nilpotent groups of class 2 and prime exponent. Journal of Symbolic Logic, 46(4):781-788, 1981.

[16] R. I. Soare. Recursively Enumerable Sets and Degrees: A Study of Computable Functions and Computably Generated Sets. Perspectives in Mathematical Logic. Springer-Verlag, Berlin, 1987. 